\title{
Graphite-Filled Composite Bipolar Plates for Fuel Cells: Material, Structure, and Performance
}

\author{
Runlin Fan ${ }^{1}$, Yuhang Peng ${ }^{1}$, Hao Tian ${ }^{1}$, Junsheng Zheng ${ }^{1,2,}$, Pingwen Ming ${ }^{1,2,{ }^{*}}$, Cunman Zhang ${ }^{1,2}$ \\ ${ }^{1}$ School of Automotive Studies, Tongji University, Shanghai 200092, China. \\ ${ }^{2}$ New Energy Automotive Engineering Center, Tongji University, Shanghai 200029, China.
}

\begin{abstract}
Bipolar plates (BPs) are one of the key components of proton exchange membrane fuel cell (PEMFC) stacks. To ensure that such a stack operates stably, a $\mathrm{BP}$ needs to meet exhibit electrical conductivity, heat conduction, $\mathrm{H}_{2}$ airtight, flexural strength, and durability. Based on these requirements, the BP should also be as thin as possible to reduce the overall cost of PEMFCs, while improving their volumetric energy density. A composite bipolar plate (CBP) exhibits the advantages of a low production cost, low processing difficulty, and corrosion resistance; it is produced using polymers and graphite as the main materials. Moreover, channel structures can be formed directly after a compression molding process. However, the trade-off that exists between electrical conductivity and flexural strength is a major challenge. The electrical conductivity of a CBP is realized through the network formed by
\end{abstract}

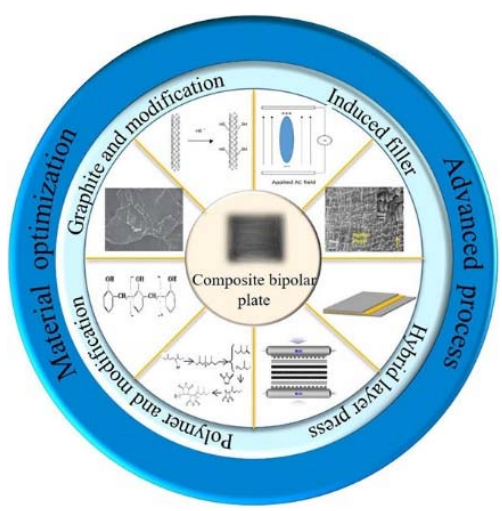
graphite materials. Therefore, it not only depends on the filler concentration, but also on the network structure. At the same time, microstructures such as accumulation polymers and graphite/resin interface are directly related to the gas tightness and flexural strength of CBP. This review summarizes the conductive fillers and polymers that are commonly used for fabricating CBPs. The universal modification methods for both (fillers and polymers) are discussed, and a brief description of the conductive theoretical model has also been included. In addition, the advanced production technology of CBP is summarized, which includes the organization of the conductive network, elimination of the polymer on the plate surface, and preparation technology of the layered plates. The relationship between the production process and the performance of the plate was also analyzed. Some studies indicate that the conductive network can be optimized by combining kinds of carbon-based filler or electric field inducing, which could significantly promote the electrical conductivity of CBP. Flexural strength and $\mathrm{H}_{2}$ permeation rates were increased by introducing carbon-based materials such as carbon fabric and graphite foil. The modification of the filler and polymer could facilitate their bonding with each other, which reduces agglomeration and increases the performance. It is worth noting that the structure had a notable influence on the performance of CBP, which was reflected in the filler/polymer interface or the hybrid layer structure. Based on this results, some ideas have been provided as the next steps that can be taken for the optimization and production of a CBP. We believe that the optimization of the CBP structure will be the key point for its future research.

Key Words: Composite bipolar plate; Electrical conductivity; Flexural strength; Production technology; Structure

Received: September 29, 2020; Revised: October 23, 2020; Accepted: October 23, 2020; Published online: November 9, 2020.

${ }^{*}$ Corresponding authors. Emails: jszheng@tongji.edu.cn (J. Z.); pwming@tongji.edu.cn (P. M.).

The project was supported by the National Key R\&D Program of China (2020YFB1505904) and the Shanghai Committee of Science and Technology, China (17DZ1200403). 


\title{
燃料电池复合石墨双极板基材的研究进展：材料、结构与性能
}

\author{
樊润林 ${ }^{1}$, 彭宇航 ${ }^{1}$, 田豪 ${ }^{1}$, 郑俊生 ${ }^{1,2,{ }^{*}}$, 明平文 ${ }^{1,2,{ }^{*}}$, 张存满 ${ }^{1,2}$ \\ 1 同济大学汽车学院, 上海 200092 \\ 2 新能源汽车工程中心, 同济大学, 上海 200092
}

\begin{abstract}
摘要: 双极板是燃料电池的重要组成部件, 需要满足导电、抗弯强度、耐腐蚀等方面性能的要求。复合石墨双极板具有成本 低、耐腐蚀性好、易成型等优势, 是双极板的一个重要研究方向。复合石墨双极板的导电功能是由以石墨为代表的导电填料 相互连接形成传导网络实现的, 抗弯强度及气密等性能则主要依靠树脂固化形成的基体。因此, 复合石墨双极板性能不仅受 到导电填料以及树脂性能的影响, 同时导电填料、树脂固化形成的结构对于极板性能也有着不可忽视的影响。本文总结了导 电填料及树脂的性质、改性方法等对于复合石墨极板性能的影响, 并分析了分子结构以及制备工艺对于极板结构以及实用性 能的影响规律。导电填料与树脂的相容性受到原料表面官能团的影响, 并直接影响了导电填料的离散均匀度以及导电填料/ 树脂的界面性能。通过填料诱导工艺优化导电网络, 能够有效提升极板的导电性能。基于对研究现状的总结, 本文对复合石 墨极板开发的未来发展方向进行了展望。
\end{abstract}

关键词: 复合双极板; 导电性; 抗弯强度; 制备工艺; 结构 中图分类号：0646

\section{1 引言}

氢能凭借着其热值高、清洁、来源广与可再生 等特点, 在清洁能源领域得到了广泛的关注 ${ }^{1-5}$ 。随 着电解制氢技术、氢气储存和运输技术的不断成 熟, 氢能可以作为太阳能、风能等的储存载体, 并 可控地转化为电能进行利用。因此, 氢能是优化能 源结构, 提高能源利用效率的重要研究领域 ${ }^{6-9}$ 。质 子交换膜燃料电池(Proton exchange membrane fuel cell, PEMFC) 能够高效地将氢气中的化学能直接 转化为电能, 其理论转化效率可以达到 $85 \%-90 \%$, 具有能量转化效率高、环境友好、室温快速启动等 特点。同时通过热电联产系统, 氢能的能量利用效 率能够进一步提升 ${ }^{10-12}$ 。

双极板 (Bipolar plates, BP) 是PEMFC中的重要 部件, 其作用主要体现在隔离与分配氧化剂和燃 料、传导电流、支撑膜电极、调节电堆内部温度等, BP在燃料电池中的装配方式如图1所示。为了满足 燃料电池的工作需求, BP必须具有优越的阻气性、 良好的导电性与耐腐蚀性、较好的导热性以及机 械性能 ${ }^{13-15}$ 。同时, 目前 BP 的重量和体积约占 PEMFC的 $80 \%$, 成本约为 $50 \%{ }^{16,17}$ 。BP在保证各项
功能性要求的前提下尽量地轻薄, 对提升PEMFC 的功率密度、降低PEMFC的生产成本有着十分重 要的意义。美国能源部(DOE)提出, 2020年BP应满 足的关键性能指标如表 1 所示 ${ }^{18}$ 。

依据基体材料, $\mathrm{BP}$ 主要可分为三类: 金属双 极板、石墨双极板以及复合石墨双极板。由于原料 的不同, 三类双极板在导电性、气密性、抗弯强度、 加工工艺等方面表现出较大的差异。

金属双极板的基体材料主要包括不锈钢、钛 合金等金属材料 19 。金属双极板具有较好的导电 性、气密性以及抗弯强度。同时由于金属具有良好 的延展性, 能够通过冲压工艺制成较薄并带有流 场结构的极板, 显著地了提升PEMFC的体积功率 密度 ${ }^{20}$ 。然而金属双极板在PEMFC环境中 $(\mathrm{pH}=2-$ $3, T=80-100^{\circ} \mathrm{C}$ )易发生腐蚀, 析出金属离子, 对 燃料电池的运行产生不良影响。同时在极板表面 形成钝化层, 导致接触电阻显著增加, 极大地影响 了金属双极板长期可靠的使用 21,22 。目前提高金属 双极板耐腐蚀性的主要工艺方案为涂覆耐腐蚀涂 层以及改进基材的耐蚀性能。添加涂层能显著改 善金属双极板的耐腐蚀性能, 但是需要兼顾其导

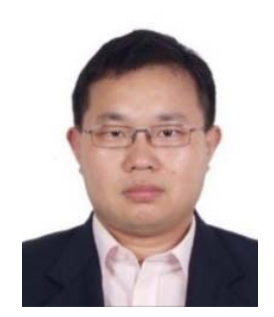

郑俊生, 1979 年生。2008 年于华东理工 大学获得博士学位。现为同济大学副研 究员。主要从事燃料电池高性能复合石 墨双极板导电机理及结构性能研究。

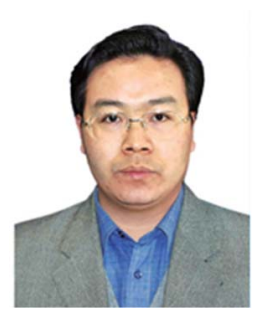

明平文, 1973 年生, 1999 年理工大学 获得博士学位。现为同济大学教授。主 要从事燃料电池的过程机理解析与衰 减抑制, 过程强化理论与超高功率密度 电堆等方面的研究。 


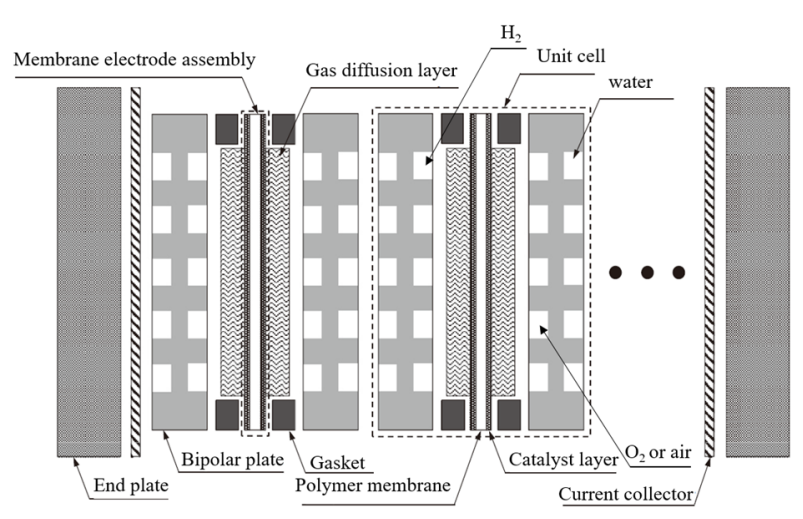

图1 PEMFC结构示意图27

Fig. 1 Schematic diagram of a PEMFC stack ${ }^{27}$.

Adapted from Elsevier Publisher.

电性能, 工艺难度以及成本偏高 ${ }^{23,24}$; 钛合金表面 的氧化膜使其具有良好的耐腐蚀性, 但同时提高 了接触电阻, 需要开发表面增导工艺 ${ }^{25,26}$ 。耐腐蚀 性问题以及工艺成本问题成为限制金属双极板大 规模推广的重要因素。

石墨是较早被开发的BP基材，具有密度低、 耐蚀性好、与气体扩散层之间接触电阻低等优点。 然而石墨双极板的孔隙率较高, 较难满足气密性 方面的要求, 同时石墨材质强度较低、质脆, 所以 成品的厚度显著高于金属双极板。关于石墨双极 板的研究多关注于对于石墨材质以及浸渍方法等 的研究, 尽管在提升气密性, 改善双极板结构强度 等方面取得了一些进展, 但是仍然不能满足在 PEMFC中的使用要求 28,29 。

复合石墨双极板是一种新型双极板, 以有机 高分子树脂及碳基导电填料为主要原料制成。其 中, 树脂基体可以增强机械性能并粘结导电填料, 是提升气密性、抗弯强度等性能的主要研究对象。 以石墨为代表的导电填料在复合材料中相互连 接, 形成传导网络, 如图2所示。复合石墨双极板导 电以及导热等功能, 主要通过传导网络来实现 ${ }^{30,31}$ 。

通过调节树脂和导电填料的成分、质量配比, 并结合石墨改性工艺或树脂添加剂, 复合石墨双 极板在的各项性能可以实现调整和优化。此外, 通 过结合先进制备工艺及后处理技术, 如对于石墨 填料在复合极板中的取向和离散形态的调整, 石 墨/树脂的界面性能的控制与优化等, 会直接影响 成型复合石墨极板中的宏观及微观结构, 进而直 接影响复合石墨极板的性能。本文总结了近年来 所开发的一系列导电填料与树脂材料, 主要包括 各类石墨材料及其改性工艺, 树脂及添加剂的使 用。并围绕着复合石墨极板结构与性能的关系, 对 复合石墨极板的先进制备工艺进行分析和讨论。
表1 2020 年DOE设定的双极板的性能目标

Table 1 DOE requirements for bipolar plate in 2020.

\begin{tabular}{cc}
\hline Properties of bipolar plate & Requirement values \\
\hline Cost & $3 \$ \cdot \mathrm{kW}{ }^{-1}$ \\
Weight & $0.4 \mathrm{~kg} \cdot \mathrm{kW}^{-1}$ \\
Hydrogen permeation coefficient & $<1.3 \times 10^{-14} \mathrm{~cm}^{3} \cdot \mathrm{s}^{-1} \cdot \mathrm{cm}^{-2} \cdot \mathrm{Pa}^{-1}$ \\
Electrical conductivity & $@ 80^{\circ} \mathrm{C}, 3.04 \times 10^{5} \mathrm{~Pa}, 100 \% \mathrm{RH}$ \\
thermal conductivity & $>100 \mathrm{~S} \cdot \mathrm{cm}^{-1}$ \\
Cathode corrosion current density & $>10 \mathrm{~W} \cdot \mathrm{m}^{-1} \cdot \mathrm{K}^{-1}$ \\
Interfacial contact resistance & $<1 \mu \mathrm{A} \cdot \mathrm{cm}^{-2}$ \\
Flexural strength & $<10 \mathrm{~m} \Omega \cdot \mathrm{cm}^{2}$ \\
\end{tabular}

\section{2 导电填料}

导电填料在复合材料中相互连接, 形成传导 网络, 是复合材料实现导电及导热功能的主要结 构, 需要具有良好的导电及导热性能。同时, 由于 导电填料的体积分数通常占 $\mathrm{BP}$ 的 $50 \%$ 以上, 需满 足极板降低成本和密度、提升极板的耐久性的目 标, 导电填料需要满足成本低, 密度低且耐酸性腐 蚀的要求。

\section{1 石墨材料}

由于石墨材料导电性和导热性好, 同时密度 较低、耐腐蚀性能好, 非常适合双极板的性能要 求。石墨材料的性质和形态直接影响着复合双极 板的导电性能, 研究表明, 与石墨填料有关, 直接 影响复合石墨双极板导电性能的因素包含：填料 的电导率、结构特性(尺寸、形状、比表面积等)、 在复合材料中填料的体积分数、分布和取向以及 石墨颗粒间填料间距等 ${ }^{33-38}$ 。不同填料结构与浓度 对于复合石墨材料导电性能的影响如图3所示。图

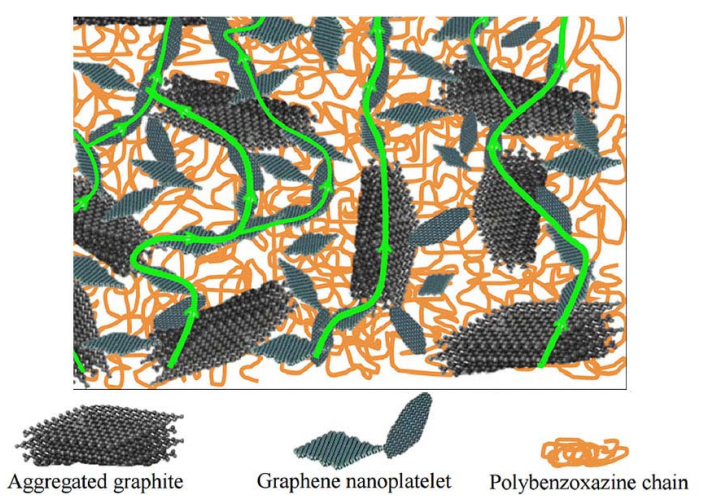

图2 导电填料在复合材料中构筑形成传导网络 32

Fig. 2 Schematic of the conductive network organized by electric filler in composite ${ }^{32}$.

Adapted from Wiley Publisher. 
3a可见, 在相同的填料含量下, 含有鳞片石墨或者 人造石墨复合材料的导电性能显著高于含有碳纤 维填料的复合材料。随着填料含量的提升, 复合石 墨材料的导电性能逐渐上升, 但是上升幅度逐渐 降低, 如图3b所示。

二维结构的石墨材料是构筑复合石墨双极板 中导电网络的一种常用导电填料, 常见的二维石 墨材料有天然鳞片石墨、膨胀石墨、石墨烯纳米片 (Graphene nanoplatelets, GNP)等 $18,32,34,37,39$ 。Petrach 等 ${ }^{40}$ 对比了不同类型石墨材料在复合石墨双极板 中的导电性能差异, 试验结果表明, 相比于一维结 构的碳纤维, 具有二维结构的石墨在复合材料中 表现出更高的导电性能。这是因为片状结构更容

(a)

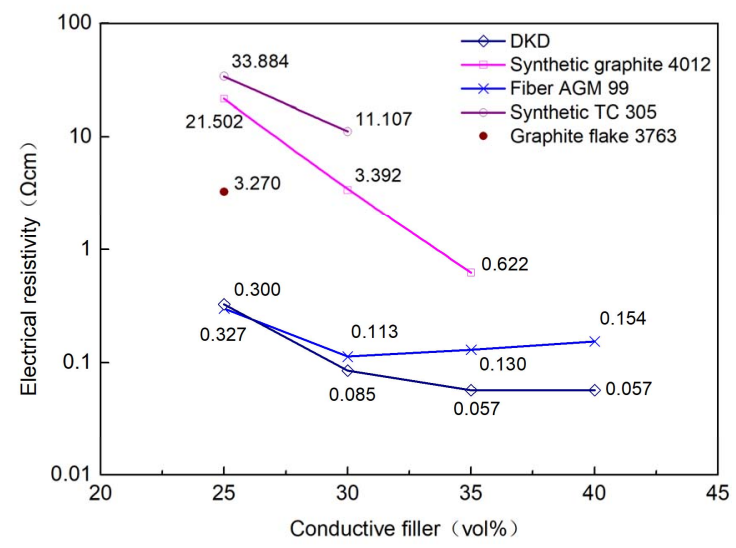

(b)

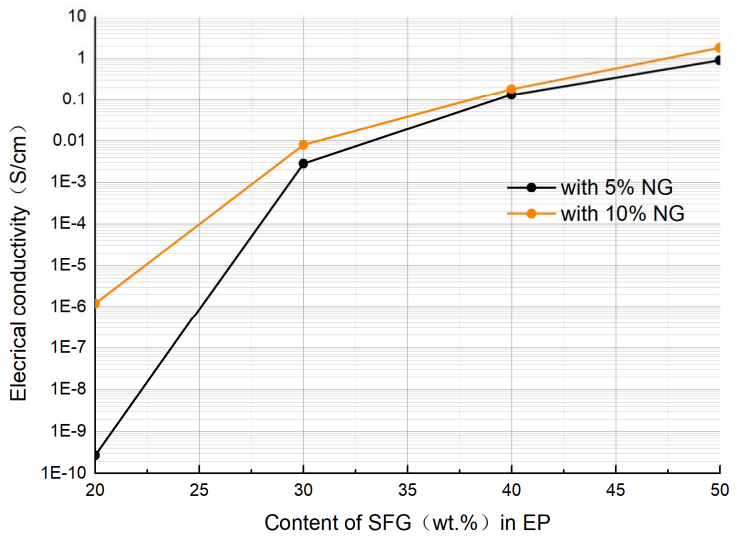

图3 填料的结构(a) ${ }^{40}$ 及浓度(b) ${ }^{41}$ 对于复合材料 导电性能的影响规律

Fig. 3 The influence of electric filler structure (a) ${ }^{40}$ and content (b) ${ }^{41}$ on in-plane electrical conductivity of composite material.

DKD, Cytec thermal graphite fibers; Fiber AGM 99: Asbury Carbons

PAN AGM 99 fiber; Synthetic graphite 4012: Synthetic graphite which diameter is $50-800 \mu \mathrm{m}$; Graphite flake 3763: natural flake which diameter is $8 \mu \mathrm{m}$; Synthetic TC 305: graphite powder. Adapted from Elsevier Publisher; Adapted from Sage Publisher.
易形成较丰富的接触面, 有利于颗粒间的电子传 递。Diaz等 ${ }^{41}$ 的研究结果显示, 随着石墨含量的增 加, 复合材料的导电性能逐渐上升, 并逐渐趋近于 极限。因此, 单纯依靠提升石墨含量, 难以满足对 于导电性能优化的目标。由于复合石墨双极板的 导电性能是通过导电填料构筑的传导通路实现 的, 因此提高导电填料的离散程度, 有利于在相近 的填料含量下达到更高的电导率。GNP作为一种 新型碳基纳米材料, 是由天然石墨经过酸性插层 工艺, 膨胀脱落后形成的超薄的石墨薄片, 具有独 特的机械及电学性能, 表面电导率可达到 $50 \times 10^{6}$ $\mathrm{S} \cdot \mathrm{cm}^{-1}{ }^{42,43}$ 。GNP与石墨的显微图像如图4所示, 可见GNP的颗粒粒径更小、更薄, 对于增强复合材 料的导电性具有显著的效果 ${ }^{44}$ 。Phuangngamphan 等 32 的研究表明, 在保持填料总量不变的情况下, GNP含量从 0 提升至 $10 \%$ 后, 复合石墨极板的电导 率从 $284 \mathrm{~S} \cdot \mathrm{cm}^{-1}$ 提升至 $329 \mathrm{~S} \cdot \mathrm{cm}^{-1}$, 且导热系数也 由 $12.5 \mathrm{~W} \cdot \mathrm{m}^{-1} \cdot \mathrm{K}^{-1}$ 提升至 $14.4 \mathrm{~W} \cdot \mathrm{m}^{-1} \cdot \mathrm{K}^{-1}$ 。

一维结构的碳基材料包括碳纤维 (Carbon fiber, CF)、碳纳米管(Carbon nanotube, CNT) 以及 多壁碳纳米管 (Multiwall carbon nanotube, MWCNT) 等 $17,38,45-47$ 。Radzuan等 ${ }^{36}$ 以 CF 为主要导 电填料, 环氧树脂为基质进行了复合石墨极板的 制备实验。结果表明, CF含量达到 $80 \%$ 的复合材料 面内电导率为 $4.26 \mathrm{~S} \cdot \mathrm{cm}^{-1}$, 贯穿电导率为 6.34 $\mathrm{S} \cdot \mathrm{cm}^{-1}$ 。添加 CNT能够有效提升复合材料的贯穿电

(a)

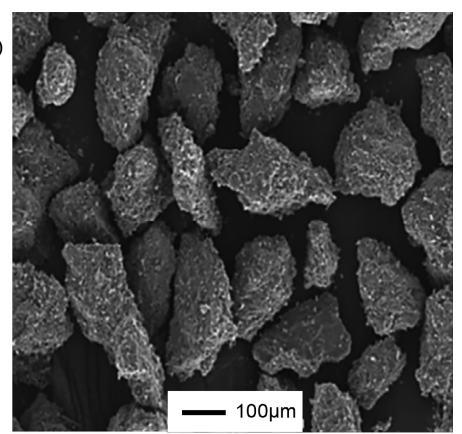

(b)

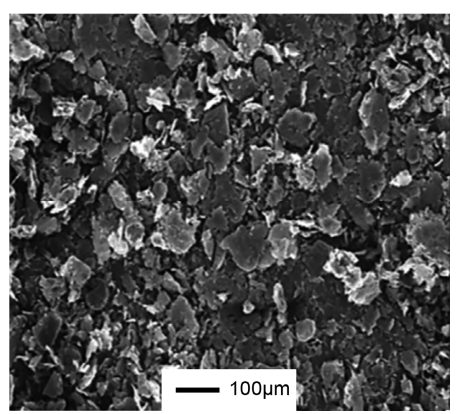

图4 石墨(a)与GNP (b) 的SEM图像 ${ }^{32}$

Fig. 4 SEM micrographs of graphite and GNP ${ }^{32}$. Adapted from Wiley Publisher. 


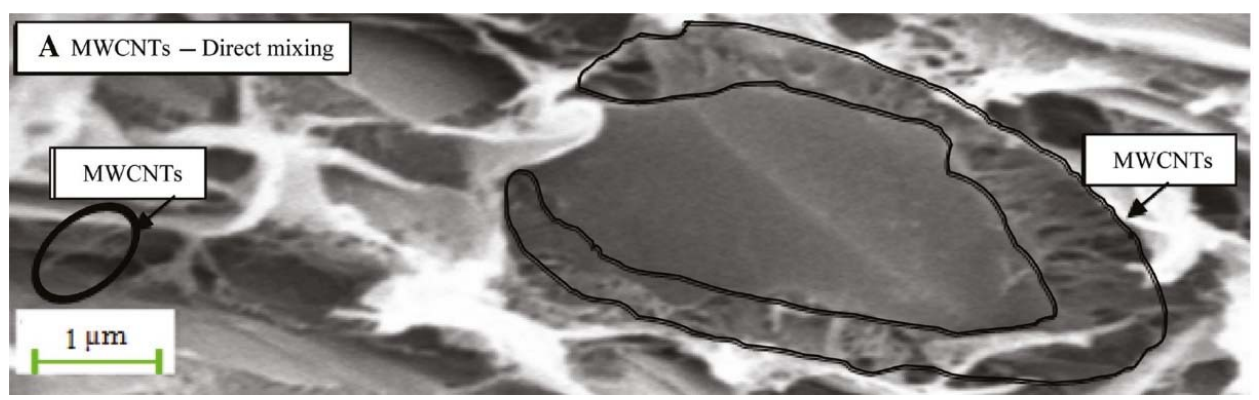

图5 MWCNT在石墨间构筑导电网络 ${ }^{34}$

Fig. 5 MWCNT linked graphite forming conductive network ${ }^{34}$.

Adapted from Walter De Gruyter Publisher.

导率, 在 CNT 含量为 $6 \%(w)$ 时, 可达到 40.31 $\mathrm{S} \cdot \mathrm{cm}^{-1}$, 但随着CNT含量的进一步提升, 复合材料 的电导率开始出现下降。Suherman等 ${ }^{38}$ 也发现了相 似的变化规律, 在CNT含量达到 $5 \%$ 时, 贯穿电导 率和面内电导率达到了最大值, 分别为 78 和 180 $\mathrm{S} \cdot \mathrm{cm}^{-1}$, 随后开始逐渐下降。Suherman等认为这与 CNT由于静电等作用发生团聚有密切的联系, 在 CNT含量较低的情况下, 可以较好地均匀离散在 复合材料中, 填充石墨颗粒间的缝隙, 提升导电性 能, 如图5所示。含量较高的情况下易发生团聚吸 附, 降低了复合材料的导电性。Wei等 48 分析认为 多种碳基材料混合在一定程度上会促进碳材料的 离散, 形成协同作用, 极大地提升了导电性能 49 。

\section{2 成型碳材料}

除石墨、CF、GNP等粉末状石墨填料外，连 续碳纤维编制体(Carbon fiber fabric, CFF)、石墨 箔、巴基纸(Buckypaper)等成型碳材料也被作为复 合石墨极板的制备材料 ${ }^{49-52}$ 。这些成型碳材料在保 留高导电性、耐腐蚀、密度低的优点之外, 凭借其 连续致密的结构, 能够有效提升复合石墨极板的 抗弯强度 ${ }^{50,53}$ 、气密性 45,54 等性能。

Hwang 等 50 以涂覆有半固化环氧树脂的 CFF, 即预浸料(Prepreg), 热模压制成带有流道结 构的复合石墨双极板。经测试, 极板的抗弯强度可 达 $159 \mathrm{MPa} 。 3 \times 10^{4} \mathrm{~Pa}$ 的压差下, 空气的气体渗透 率为 $1.4 \times 10^{-6} \mathrm{~cm}^{3} \cdot \mathrm{cm}^{-2} \cdot \mathrm{s}^{-1}$, 表现出了优化复合石 墨极板机械性能的巨大潜力。

Kang 等 ${ }^{51}$ 以单向碳纤维预浸料作为中间层进 行了复合石墨极板的制作, 并利用天然石墨粉涂 敷在预浸料表面, 降低复合极板的面积比电阻 (Area specific resistance, ASR)。经测试, 利用预 浸料可完成厚度为 $1.6 \mathrm{~mm}$ 的复合极板, 并形成深 度为 $1 \mathrm{~mm}$ 的交错式流道, 如图6所示。可见预浸料 的使用有利于超薄复合石墨极板的成型。此外, 该 极板的面内电导率最大值为 $170 \mathrm{~S} \cdot \mathrm{cm}^{-1}$, 贯穿电导
率最大值为 $38 \mathrm{~S} \cdot \mathrm{cm}^{-1}$, 满足DOE对于极板导电性 能的要求。

巴基纸(Buckypaper)是一种由缠结的 CNT网 络生成的自支撑且自立的薄片材料, 具有超强强 度和高导电性 ${ }^{55}$ 。Yao 等 ${ }^{56}$ 利用巴基纸进行了复合 石墨极板的制备。试验结果显示, 巴基纸可小幅提 升复合石墨极板的抗弯强度, 但未能表现出理论 情况下对于导电性能的提升。这是由于成型过程 中树脂发生了自由流动, 包覆在巴基纸表面, 形成 了高电阻层, 影响了对于巴基纸对导电性能的提 升。 $\mathrm{Yu}$ 等 ${ }^{49}$ 将石墨䈃覆盖在复合材料表面, 并进行 热模压成型, 成功地将ASR降低了 $80 \%$ 以上。他们 发现, 在热压成型过程中, 石墨䈃能够有效将树脂 吸收, 避免树脂聚集, 同时导电结构没有受到树脂 的影响。

综上所述，成型碳材料能够对复合石墨极板 机械性能、气密性、导电性能的优化, 对于开发高 性能极板具有重要价值。但是由于延展性能不足, 不能满足流道结构的精细成型要求, 仍需要以石

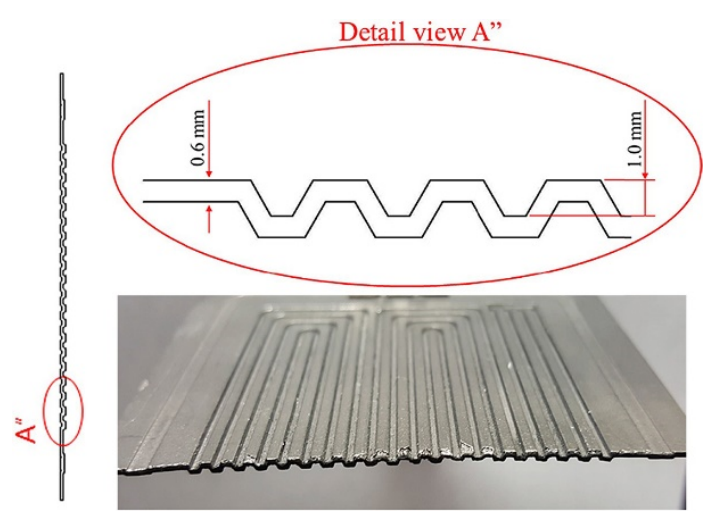

图6 预浸料制备带有流道结构的复合石墨极板截面 ${ }^{51}$

Fig. 6 The cross-sectional view of corrugated composite flow field plate made with the graphite-prepreg hybrid layer ${ }^{51}$.

Adapted from Elsevier Publisher. 
墨/树脂复合材料完成高精度流道结构的成型 51 。

\section{3 导电材料的改性处理}

碳基材料的表面特性使得树脂与石墨之间通 常不能紧密地粘合, 且小粒径颗粒更易发生团聚, 均匀离散难度较高 ${ }^{57,58}$ 。通过化学改性引入新的官 能团, 能有效提升填料与树脂的界面性能, 进而实 现复合石墨极板性能的提升。Yin等 ${ }^{59}$ 利用Fenton 法对CNT进行氧化处理, 在 CNT表面形成了羟基 官能团和羧基官能团。研究表明, 经过Fenton法对 $\mathrm{CNT}$ 氧化后, 复合材料的抗弯强度从 $61.6 \mathrm{MPa}$ 提升 至68.6 $\mathrm{MPa}$, 导电率从 $141.6 \mathrm{~S} \cdot \mathrm{cm}^{-1}$ 提升至 145.2 $\mathrm{S} \cdot \mathrm{cm}^{-1}$ 。通过氧化处理后, 碳基材料的极性官能团 能够与树脂支链的官能团相结合, 如图7所示。经 过氧化后, 在 CNT表面形成了羟基 $(-\mathrm{OH})$ 及羧基 $(-\mathrm{COOH})$, 如图7a所示。在成型过程中, CNT表 面的羟基与 $\mathrm{PF}$ 表面的官能团形成化学键, 如图 $7 \mathrm{~b}$ 所示。通过化学键的结合, 提高了石墨/树脂的相 容性, 有利于复合石墨性能的提升。

Athmouni等 ${ }^{60}$ 利用硝酸对 MWCNT进行官能 团化处理, 并将MWCNT离散在聚对苯二甲酸丁二 醇酯(Polybutylene terephthalate, PBT)中。官能团 化后的MWCNT与PBT有着良好的界面相互作用 和相容性。经官能团化的MWCNT能显著降低复合 材料的 $\operatorname{ASR}\left(0.25 \Omega \cdot \mathrm{cm}^{2}\right.$ 下降至 $\left.0.13 \Omega \cdot \mathrm{cm}^{2}\right)$, 并提升 了其抗弯强度 $(9.2 \mathrm{MPa}$ 提升至 $10.8 \mathrm{MPa})$ 。在PBT中 离散后的MWCNT能够进一步提升极板性能, ASR 下降至 $0.08 \Omega \cdot \mathrm{cm}^{2}$, 抗弯强度提升至 $13.4 \mathrm{MPa}$ 。此 外, 提升石墨与树脂基质相容性的改性工艺还有 对石墨进行氟改性 ${ }^{61}$, 聚氧化烯-胺改性 ${ }^{62}$ 等工艺方 法。通过在石墨中枝接官能团, 能够有效地提升石 墨与树脂的相容性, 成为提升石墨/热塑性树脂复 合材料性能的一个重要研究方向。

对石墨的改性处理通常还包括对于石墨导电

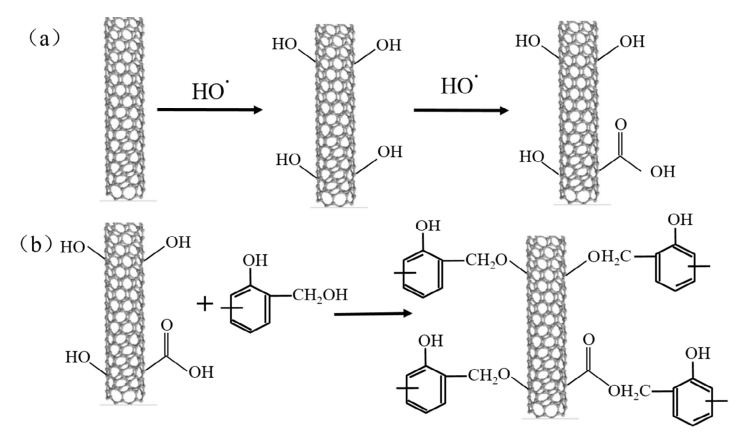

图7 CNT的氧化处理(a)及其与 PF结合(b)示意图 59

Fig. 7 Schematic of Fenton oxidized reaction of CNT (a) and combination between treated CNT and PF (b) ${ }^{59}$.

Adapted from Elsevier Publisher.
性能的提升。 $\mathrm{Li}$ 等 ${ }^{63}$ 对 GNP进行气相溴化处理, $\mathrm{Br}$ 插入 GNP的石墨烯层间, 形成新的离子键, 能够有 效提升复合石墨材料的导电性能。Matsumoto等 ${ }^{64}$ 开发出了金属氯化物掺杂石墨的工艺路线, 通过 在石墨层间掺杂两种金属氯化物如 $\mathrm{AlCl}_{3}-\mathrm{FeCl}_{3}$ 或 $\mathrm{AlCl}_{3}-\mathrm{CuCl}_{3}$, 能够有效提升石墨材料的导电性能。

\section{4 导电理论模型}

对于导电原理的研究也是复合石墨极板研究 的重点之一。基于渗滤理论对于填料颗粒间导电 行为的理论解释, 结合颗粒的形态、电导率、含量 等因素的影响, 逐渐形成了描述复合石墨材料导 电性能的理论模型, 对提升复合石墨极板的导电 性能优化提供了理论指导 $31,36,65-67$ 。主要的导电理 论模型包括了: (1)基于渗流理论与颗粒相互接触 的概率计算形成的统计学模型 ${ }^{68}$; (2) 考虑填料与聚 合物的表面能与熔融粘度提出的热力学模型 ${ }^{69}$; (3) 研究填料与树脂的导电性能以及形貌对导电性能 影响的几何学模型; (4)研究填料的形貌结构与取 向的结构学模型70等。

目前认可度较高的导电理论模型为几何学模 型中的通用有效介质模型 (General Effective Media, GEM), 通过将填料的体积分数、形状及大 小、纵横比、临界渗滤阈值等因素纳入模型中, 能 够较为准确的分析含有一种或多种导电填料, 以 及高导电填料含量情况下, 复合石墨材料的导电 性能 $31,36,67$ 。以含有石墨 $(\mathrm{G})$ 与 $\mathrm{CF}$ 的复合材料为例, GEM模型如式(1)与式(2)所示:

$$
\frac{\left(1-\emptyset_{\mathrm{G}}-\emptyset_{\mathrm{CF}}\right)\left(\sigma_{\mathrm{EP}}^{\frac{1}{t}}-\sigma_{m}^{\frac{1}{t}}\right)}{A=\frac{\frac{1}{\sigma_{c}}}{\sigma_{\mathrm{EP}}^{t}+A \sigma_{m}^{\frac{1}{t}}}}+\frac{\emptyset_{\mathrm{G}}\left(\sigma_{\mathrm{G}}^{\frac{1}{t}}-\sigma_{m}^{\frac{1}{t}}\right)}{\sigma_{\mathrm{G}}^{\frac{1}{t}}+A \sigma_{m}^{\frac{1}{t}}}+\frac{\emptyset_{\mathrm{CF}}\left(\sigma_{\mathrm{CF}}^{\frac{1}{t}}-\sigma_{m}^{\frac{1}{t}}\right)}{\sigma_{\mathrm{CF}}^{\frac{1}{t}}+A \sigma_{m}^{\frac{1}{t}}}=0
$$

其中, $\sigma_{m}$ 是复合材料的电导率; $\sigma_{\mathrm{EP}}$ 是聚合物的电 导率; 而 $\sigma_{\mathrm{G}} 、 \sigma_{\mathrm{CF}}$ 是导电填料的电导率; $t$ 是指数成 分, 是体现填料形态对于导电性能影响的参数; $A$ 是复合物的渗滤阈值; $\phi_{\mathrm{G}} 、 \phi_{\mathrm{CF}}$ 是填料的体积分数; $\phi_{c}$ 是渗滤阈值下的填料体积分数。但是 GEM模型 是基于填料均匀混合的理想条件下，对于复合石 墨极板导电性能的预测, 未能将填料的取向和分 布对于导电性能的影响纳入模型中。

\section{3 树脂材料}

目前用于复合石墨双极板的树脂材料依据成 型特性可以区分为热固性树脂和热塑性树脂。由 于分子结构以及成型特性的不同, 以两种类型的 树脂为基础的复合材料在成型工艺以及性能等方 面表现出了较为明显的差异。 


\section{1 热固性树脂及改性工艺}

热固性树脂是目前在复合石墨极板的研发制 备中应用较为广泛的一类树脂, 其分子链中通常 具有较多的活性基团, 在成型过程中相互交联, 形 成稳定的分子结构。常见的有环氧树脂(Epoxy, EP)、酚醛树脂(Phenolic resin, PF)、乙烯基酯树脂 (Vinyl ester resin, VE)、聚苯并噁嗪 (Polybenzoxazine, PBA) 等。热固性树脂具有良好 的耐热性, 可以与石墨形成更可靠的界面, 通常具 有更高的抗弯强度, 允许极板较薄的成型厚度。

Akhtar等 ${ }^{35}$ 以EP作为基体, 使用联胺作为固化 剂, 天然石墨和MWCNT作为导电填料制备复合石 墨极板, 并分析复合极板在不同温度条件下的性 能差异。实验结果表明, 随着温度从 $25^{\circ} \mathrm{C}$ 提升到 $200^{\circ} \mathrm{C}$, 弯曲强度和肖氏硬度逐渐下降, 但是面内 和通面电导率均有所上升。为了提升 $\mathrm{EP}$ 的高温性 能, Lee等 ${ }^{71}$ 使用氰酸酯对EP进行改性处理, 提高 了EP的玻璃化转变温度, 同时保持了EP的机械性 能不发生明显改变。

欧阳涛等 72 以 $\mathrm{PF}$ 与膨胀石墨混合, 通过热压制 成复合石墨极板, 并使用聚乙烯醇缩丁醛 (Polyvinyl butyral, PVB) 对PF进行改性优化。试验 结果显示, 当 $\mathrm{PVB}$ 与 $\mathrm{PF}$ 的含量接近 $1: 2$ 时, 复合石 墨极板的电导率和抗弯强度达到最大值, 较单独 使用 PF 分别提升 $12.3 \%$ 、 $14.2 \%$ ，达到了 192.3 $\mathrm{S} \cdot \mathrm{cm}^{-1}, 47.25 \mathrm{MPa}$ 。

Phuangngamphan等 ${ }^{32}$ 认为PBA在热固化的过 程中表现出较高的流动性, 能够有效地在成型过 程中与填料进行润湿和结合。同时PBA本身具有 良好的机械强度与弹性模量, 是一种适合复合石 墨极板开发的热固性树脂。由于PBA良好的润湿 性, 能够允许填料的质量分数达到 $80 \%$ 以上, 并实 现了电导率达到 $323 \mathrm{~S} \cdot \mathrm{cm}^{-1}$, 导热率为 14.5 $\mathrm{W} \cdot \mathrm{m}^{-1} \cdot \mathrm{K}^{-1}$, 抗弯强度为 $55 \mathrm{MPa}$ 的良好性能。

\section{2 热塑性树脂及改性方法}

热塑性树脂由大量线状有机高分子组成, 若 分子进行规则排列则会形成结晶, 分子排列越规 则, 则结晶度越高。热塑性树脂在温度升至熔点温 度 $\left(T_{\mathrm{m}}\right)$ 时, 会逐渐软化甚至融化, 温度降低后凝固 成固体。结晶度不同, 融化过程也不尽相同。此外, 随着温度的升高, 热塑性树脂会从硬且脆的玻璃 态转变为具有较高弹性的橡胶态, 发生这一转变 的温度被称为玻璃转移温度 $\left(T_{\mathrm{g}}\right)$ 。因此, 不同种类 的热塑性树脂的各种特征温度是分析其使用性能 的重要依据。表2展示了热塑性复合材料中较为常 见的热塑性聚合物的物理性质 ${ }^{73}$ 。由表 2 可见, 高 密度聚乙烯(High Density Polyethylene, HDPE)、 聚丙烯 (Polypropylene, PP) 、聚偏氟乙烯 (Poly(vinylidene fluoride), PVDF)等由于热变形温 度(Heat deflection temperature, HDT)较低, 不适用 于高温燃料电池。聚醚酰亚胺 (Polyetherimide, PEI)、聚苯硫醚(Polyphenylene sulfide, PPS)等热 变形温度较高, 较适宜用作高温燃料电池的复合 石墨双极板。

由于 PP具有相对较低的成本, 并易于加工, 是较早运用于制作复合石墨双极板的热塑性树 脂 74 。由于热塑性树脂的成型特点, PP可以与石墨 进行熔融混合并完成注塑定型。Yovial等 ${ }^{75}$ 分析了 石墨填料的含量及形态对于熔融混合后复合材料 工艺性能的影响规律。结果显示, 随着石墨尺寸的 减小和含量的增加, 注塑导电复合材料的流动性 降低。这与颗粒间相互吸引后形成集聚团有直接 的关联。由于PP分子中缺少极性官能团, 不利于 石墨填料在PP中的均匀分散, PP与石墨的界面性 能也不理想。其他类型的热塑性树脂也表现出了 相似的问题76-78。

为了提升石墨在热塑性树脂中的离散度, Liao等 79 认为结晶度对热塑性树脂与石墨的混合

表2 常见热塑性树脂的性能对比 ${ }^{73}$

Table 2 The properties of the commonly used thermoplastic polymers ${ }^{73}$.

\begin{tabular}{|c|c|c|c|c|c|c|c|}
\hline Polymer & Density $/\left(\mathrm{g} \cdot \mathrm{cm}^{-3}\right)$ & $T_{\mathrm{g}} /{ }^{\circ} \mathrm{C}$ & $T_{\mathrm{m}} /{ }^{\circ} \mathrm{C}$ & $T_{\text {process }} /{ }^{\circ} \mathrm{C}$ & $\mathrm{HDT} /{ }^{\circ} \mathrm{C}$ & Flexibility/GPa & Shrinkage $/ \%$ \\
\hline LDPE & $0.917-0.94$ & -110 & 115 & $180-240$ & $40-50$ & $0.245-0.33$ & $2-4$ \\
\hline HDPE & $0.94-0.97$ & -110 & 135 & $200-300$ & $60-90$ & $0.75-1.575$ & $1.5-4$ \\
\hline PP & $0.9-0.91$ & -10 & 170 & $180-300$ & $100-120$ & $1.2-1.6$ & $1-3$ \\
\hline PET & $1.3-1.4$ & $73-78$ & 260 & $270-290$ & $75-115$ & $2.8-3.5$ & $0.2-3$ \\
\hline PEI & $1.27-1.3$ & 215 & 370 & $370-400$ & $195-210$ & $3-3.4$ & $0.7-0.8$ \\
\hline PEEK & $1.26-1.32$ & 145 & 343 & $370-420$ & 150-160(@1.8 MPa) & $3.7-4$ & $1.2-1.5$ \\
\hline PVDF & $1.7-1.8$ & $-42--25$ & 177 & $190-280$ & $70-150$ & $1.5-2$ & $2-4$ \\
\hline PPS & 1.35 & $88-93$ & 280 & $300-350$ & $140-160$ & $3.8-4.2$ & $0.6-1.4$ \\
\hline
\end{tabular}


有着直接的影响。石墨在低结晶度的基质中的分 散度更高, 进而表现出了更高的电导率、机械性能 和热稳定性。Adloo等 ${ }^{80}$ 通过具有较多极性基团的 马来酸䣶(Maleic anhydride, MAH)对PP进行枝接, 有效提升了石墨在PP中的相容性。相比于没有枝 接的复合材料, 抗弯强度提升至 $44.28 \mathrm{MPa}$, 电导 率从 $11.4 \mathrm{~S} \cdot \mathrm{cm}^{-1}$ 提升至 $104.63 \mathrm{~S} \cdot \mathrm{cm}^{-1}$ 。图 8 为 $\mathrm{MAH}$ 枝接PP前后复合材料的电镜分析图像。图8a中有 着大量由于石墨颗粒团聚形成的黑色斑点, 图 $8 \mathrm{~b}$ 中的黑色斑点密度明显降低, 表明由于石墨颗粒 离散度的提升, 石墨颗粒的团聚现象有了明显的 改善。Caglar等 ${ }^{81}$ 利用钛酸酯偶联剂对PPS进行改 性, 随后与石墨填料混合。试验结果显示, 偶联剂 的加入能够有效降低熔融态复合材料的表观粘 度, 提升其流动性能。开发新型热塑性树脂也是提 升石墨在树脂中分散度的重要方法。Lee等 ${ }^{82}$ 的研 究表明, 在氟化乙烯-丙烯(Fluoronated ethylenepropylene, FEP)基质中, 导电填料能够实现较好的 分散, 并通过熔融混合和挤压注塑实现了对导电 填料的定向。制备形成的复合材料表现出优异的 导电性能和机械性能。

为了平衡过高石墨含量导致的导电性与抗弯 强度矛盾。Hopmann等 83 利用EPDM橡胶提升PP的

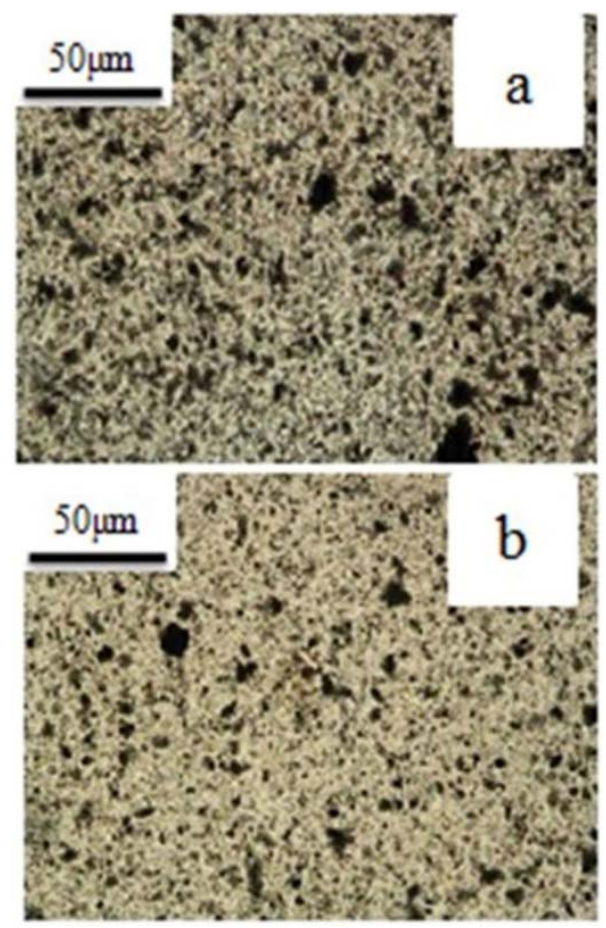

图8 MAH枝接PP前(a)后(b)复合材料的截面SEM图像 ${ }^{80}$

Fig. 8 SEM images of cross-sectional view of the composites before(a) and after(b) mixing MAH in PP ${ }^{80}$.

Adapted from Elsevier Publisher.
抗冲击改性剂, 测试结果表明, EPDM橡胶会使得 复合材料的机械强度显著提升, 但会导致导热系 数出现小幅下降。同样, 导电聚合物, 如聚吡咯 (Polypyrrole, PPy), 聚苯胺(Polyaniline, PANi)和 聚噻吩也可用于生产导电复合材料 $73,77,84$ 。然而由 于PANi的热稳定性不佳, 会出现加热制备过程中 受热分解的情况, 对加工工艺有较高的要求 ${ }^{74}$ 。

为了充分利用不同复合材料的性能优势, 部 分研究者开发出了热塑性树脂与热固性树脂混合 使用的工艺思路。Alo等 ${ }^{85}$ 将马来酸酐枝接的聚丙 烯(MAH-g-PP)、PP、石墨粉 $190{ }^{\circ} \mathrm{C}$ 熔融混合, 然 后依次加入环氧树脂与固化剂。形成的粉料通过 热模压成型, 制成复合石墨极板。实验结果表明, 在MAH-g-PP的辅助下, PP与EP表现出良好的相 容性, 在石墨的填充质量分数达到 $70 \%$ 时, 复合材 料的抗弯强度达到 $55 \mathrm{MPa}$, 通面电导率为 1.91 $\mathrm{S} \cdot \mathrm{cm}^{-1}$, 面内电导率为 $55.24 \mathrm{~S} \cdot \mathrm{cm}^{-1}$, 均表现出优于 单独使用 $E P$ 或PP的性能。

\section{4 极板结构与制备工艺}

复合石墨极板的研究在初期主要集中在对于 高性能材料的开发以及通过材料改性实现复合石 墨极板某方面性能的提升。随着研究的逐渐深入, 传导网络对于复合石墨极板性能的影响已经得到 了较为普遍的认同, 并基于优化传导网络的目标 开展了多项研究工作。不仅通过添加纳米导电颗 粒丰富传导网络, 同时注重在材料成型过程中, 对 于导电填料在复合材料中分布和取向的干预和控 制。同时复合材料中的微观结构对于复合石墨极 板性能的影响规律也开始得到了广泛的关注。为 了实现对极板结构的控制和优化, 新的制备工艺 得到了开发, 对提升复合石墨极板的性能发挥了 独特的作用。

\section{1 复合石墨材料的混合及成型}

复合石墨极板通常是由石墨与树脂经过充分 混合后, 形成母料(Master batch), 然后填入模具中 经过热压定型制成的。为了保证复合石墨极板可 靠成型且性能可靠, 通常需要将石墨与树脂进行 充分均匀的混合, 而混合的均匀度与混合条件有 着直接联系 ${ }^{41}$ 。

Wei等 ${ }^{48}$ 将填料与EP分别溶解在丙酮中, 经超 声处理及高速摚拌后, 放入真空干燥箱中 $80^{\circ} \mathrm{C}$ 干 燥, 将丙酮溶剂完全去除, 粉碎后即得到了均匀混 合的母料, 随后母料放入模具中加热固化得到成 型的极板。以溶剂为载体的湿法混合能保证树脂 与石墨实现充分的均匀混合, 但是干燥时间达到 5 
$\mathrm{h}$, 影响生产效率。Simaafrookhteh等 ${ }^{86}$ 采用含有固 化剂的PF粉末，与鳞片石墨通过机械搅拌进行干 法混合制备成母料。随后将母料放入预成型模具 中, 以 $175^{\circ} \mathrm{C} 、 120 \mathrm{MPa}$ 的压力压缩 $2 \mathrm{~min}$, 实现预 成型。将预成型的板材转移到定型模具中, $180^{\circ} \mathrm{C}$ 、 $120 \mathrm{MPa}$ 的压力保持 $1 \mathrm{~min}$, 实现复合石墨材料的 成型, 表现出了良好的生产效率。当复合材料中具 有多种填料时, 为了保证混合均匀度, 通常首先对 多种填料进行球磨混合, 同时对树脂与固化剂进 行快速混合, 随后将填料与固化剂在内部混合器 中进行约 $10 \mathrm{~min}$ 的混合, 完成母料的制备 ${ }^{35,38}$ 。

热塑性树脂通常呈现为颗粒或粉末状的形 式, 因此混合过程与热固性树脂表现出明显的差 异。由于 CNT易发生团聚, Park等 ${ }^{87}$ 利用乙醇作为 离散剂, 将CNT与FEP粉末进行充分混合离散, 经 过干燥后得到CNT@FEP混合料, 随后与石墨进行 球磨混合, 得到均匀混合的母料。在模具中以 $30{ }^{\circ} \mathrm{C} 、 13.79 \mathrm{MPa}$ 成型条件下成型 $1 \mathrm{~h}$, 得到复合 石墨极板。经过充分离散的CNT能够更有利于促 进复合石墨极板导电性能与机械性能的提升。 Liao等 88 则利用 $29000 \mathrm{r} \cdot \mathrm{min}^{-1}$ 的超高速机械摚拌实 现石墨烯、石墨以及碳纤维的混合以及导电填料 与聚乙烯粉末的混合。利用热塑性树脂能够反复 成型的特点, 树脂与导电填料的混合可以通过加 热熔融混合来实现。Kim等 89 将PPS粉末与 GNP球 磨混合后对混合物进行了 $320^{\circ} \mathrm{C} 、 30 \mathrm{~min}$ 的熔融混 合, 冷却后粉碎, 得到母料。随后将母料与PPS粉 末以及石墨粉球磨混合, 将混合好的粉料放入模 具中以 $300^{\circ} \mathrm{C} 、 13.79 \mathrm{MPa}$ 的压力热压成型 $1 \mathrm{~h}$ 得到 成型极板。同时Kim等还制备了未经熔融混合, 仅 通过球磨完成PPS、GNP以及石墨混合得到的极 板, 与经过熔融混合得到的极板进行了性能对比。 研究结果显示, 熔融混合更有利于 GNP在复合材 料中的均匀离散, 极板的电导率和热导率均有了 显著提升。此外, 他们还提出, 为了追求较高的电 导率, 复合石墨材料的碳含量较高, 热塑性复合材 料熔融状态下流动性较差, 不能通过注塑成型实 现复合石墨极板成型。Mahyoedin等 ${ }^{75}$ 也发现了相 似的现象。因此, 热压成型几乎是高石墨含量复合 材料最终定型最主要的方法。

\section{2 导电填料的定向工艺}

通过调整生产加工工艺, 在成型过程中对导 电填料的取向和分布进行调节和控制, 能够显著 影响复合石墨极板的导电性能。Radzuan等 ${ }^{90}$ 将研 磨后的CF、CNT与PP进行熔融混合, 然后通过挤 出工艺实现混合物中的 $\mathrm{CF}$ 与 $\mathrm{CNT}$ 的定向, 通过热
模压成型制作成型极板。其断面的SEM图像如图9 所示, 经过挤出工艺后的极板中的 $\mathrm{CF}$ 呈现规则排 列。研究结果表明, 沿纤维定向排列方向的电导率 远大于其他方向, 充分表明导电网络的有序组织 对于提升极板的导电性能有着重要的意义。其次, 填料的取向对于复合材料的机械性能也有着直接 的影响, 在 $\mathrm{CF}$ 含量为 $65 \%, \mathrm{PP}$ 含量为 $30 \%$ 的情况 下, 抗弯强度可以达到 $143.37 \mathrm{MPa}$ 。Huang 等 ${ }^{91}$ 的 研究结果也证实了填料的取向对于复合材料的机 械性能有着重要的影响。

Martin等92将MWCNT离散在EP溶液中, 并对 比了直流电场(direct-current electric field, DC)与交 流电场(alternating electric field, AC) 诱导填料分布 效果。经过电场诱导后, 填料呈现出沿电场方向有 序分布的趋势, 同时沿该方向的电导率显著上升。 然而在DC的作用下, 填料由于电泳作用趋向于富 集在极板表面, 不利于填料在基质中的均匀分布, 如图10a所示。而图10b中填料更均匀地分布在基 质中, 形成了更丰富的导电网络。因此 $\mathrm{AC}$ 更适用 于复合材料中的填料诱导工艺。Evangelos 等 ${ }^{93}$ 利 用 EP对单向碳纤维编织布进行增强, 并利用 $\mathrm{AC}$ 实 现对离散在EP中氧化石墨烯(GO)的取向控制。试验 结果表明, 经电场进行取向控制形成的贯穿电导 率与热导率显著高于随机取向形成的复合材料, 且碳纤维和定向 $\mathrm{GO}$ 的组合使得成型板材在平面 和厚度方向均表现出良好的导电性。Evangelos 等 ${ }^{93}$ 认为电场辅助填料校准工艺仅需 $30 \mathrm{~V} \cdot \mathrm{mm}^{-1}$ 的电 场强度即可实现, 适宜工业生产应用。Gupta等 ${ }^{94}$ 将 $220 \mathrm{~V}$ 交流电场与脉冲电流相结合使用, 对离散 在PVDF中的CNT定向。试验结果表明, AC仅可以 实现CNT的定向排列, 不能促使CNT相互接触, 电 导率仅提升了 $28 \%$, 如图 11a所示。脉冲电流有利 于促进CNT相互接触, 且使填料的排列更加规则, 电导率提升了 $360 \%$, 如图 $11 \mathrm{~b}$ 所示。Wang 等 95 的研 究也表明, CNT定向排列后, 不仅有利于提升导电

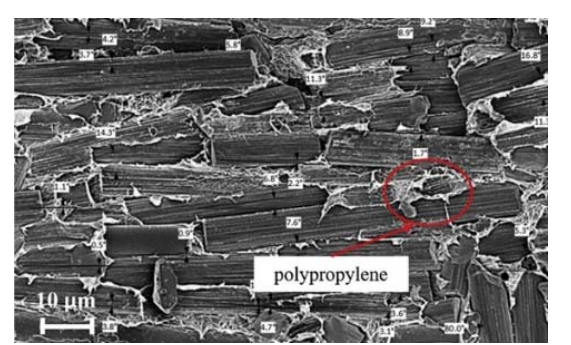

图9 熔融混合挤出后复合材料的显微图像 ${ }^{90}$

Fig. 9 SEM image of composite prepared by melt mixed and extruded ${ }^{90}$. Adapted from Elsevier Publisher. 
(a)

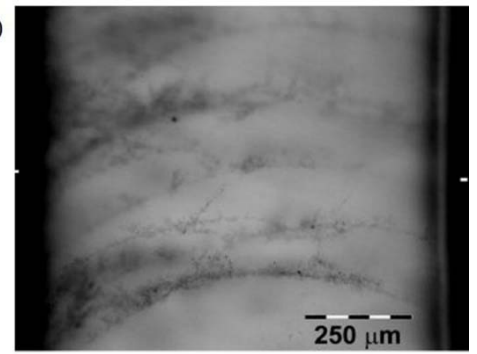

(b)

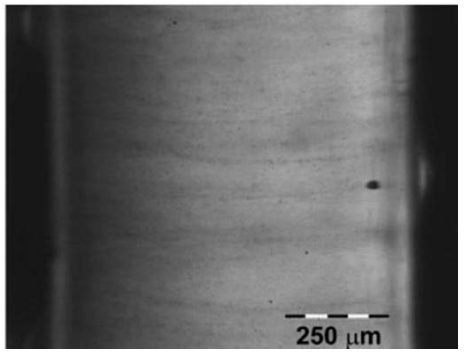

图10 DC (a)与 $A C$ (b) 定向后的MWCNT分布差异 ${ }^{92}$

Fig. 10 Comparison of the MWCNT induced by $\mathrm{DC}$ (a) and $\mathrm{AC}(\mathrm{b}){ }^{92}$. Adapted from Elsevier Publisher.

(a)

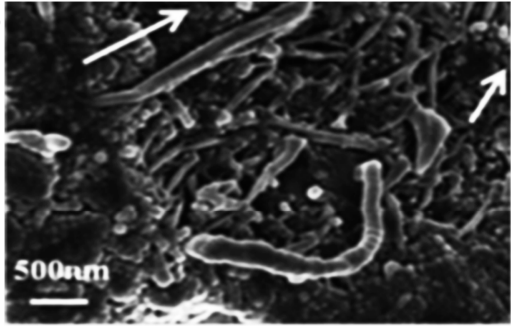

(b)

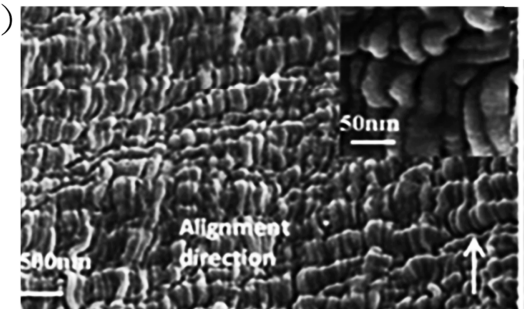

图11 AC定向CNT的分布取向对比, (a)无脉冲电流;

(b)施加脉冲电流 ${ }^{4}$

Fig. 11 Comparison of distribution and orientation of

CNT induced by AC (a) without pulse current; (b) applied pulse current ${ }^{94}$. Adapted from Elsevier Publisher.

性能，同时抗弯强度也有着显著的增强。复合材料 中导电填料的取向控制方法还包括磁场 ${ }^{96,97}$ 、静电 纺丝 $98-100$ 等技术。然而目前的有关研究均仅涉及 质量分数在 $5 \%$ 下 $\mathrm{CNT} 、 \mathrm{GO}$ 等高导电纳米颗粒的定 向工艺, 较少涉及到对于碳纤维、石墨颗粒等的定 向处理。因此, 交流电场、磁场以及静电纺织等技 术适用于复合石墨极板预制层的制备过程。关于 复合石墨极板预制层的研究和讨论将在4.4节进行
讨论。

\section{3 微观结构与性能优化}

复合石墨极板的性能不仅取决于石墨、树脂 材料的本征性能, 极板的微观结构对于极板的性 能也有着直接的影响。不仅体现在传导网络的组 织, 石墨/树脂的界面性能、树脂的积聚等微观结 构也会显著影响极板的性能。

Avasarala等 101 发现在复合材料的成型过程 中, 树脂会富集在极板表面, 导致极板与气体扩散 层之间出现了较高的接触电阻。巴基纸表面具有 着丰富的网状结构, 如图12a所示。Yao等52在试验 中将巴基纸包覆在母料表面进行热压成型，由于 树脂在成型过程中发生流动，极板表面的巴基纸 被树脂包覆，导致接触电阻明显升高，如图 $12 \mathrm{~b}$ 所 示。因此，去除极板表面富含树脂的区域，将导电 填料充分暴露出来, 有利于降低极板的接触面电 阻。

目前公开了多种技术去除极板表面的富集树 脂, Lee等 102,103 将FEP薄膜、聚酯纤维薄膜等有机 高分子薄膜铺设在复合材料与模具之间。在热模 压成型过程中，有机高分子薄膜能够有效吸收流 动到极板表面的EP，在成型完成后将有机高分子 从极板表面剥离。试验结果表明, 通过对树脂的吸 附, 极板的面积比电阻(Area specific resistance, ASR)能够从 $122.9 \mathrm{~m} \Omega \cdot \mathrm{cm}^{2}$ 下降至 $26.3 \mathrm{~m} \Omega \cdot \mathrm{cm}^{2}$, 同 时极板强度提升了 $22 \%$ 。同时极板的气密性并不会 因为树脂被吸附而显著降低。Yu等 ${ }^{104}$ 利用等离子 体对压制成型的复合石墨极板进行表面处理，氧

(a)

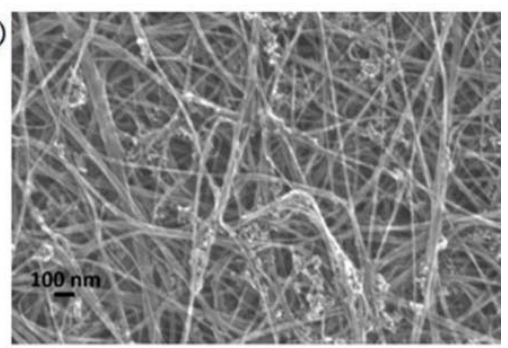

(b)

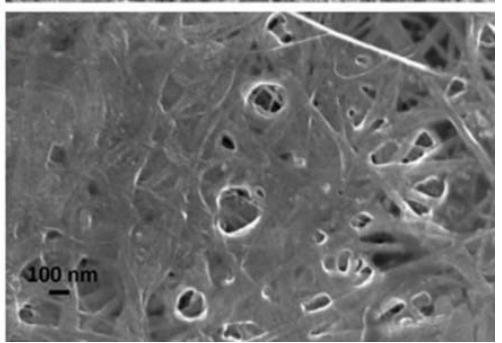

图12 模压成型前(a)以及成型后(b)巴基纸的SEM图像 ${ }^{52}$

Fig. 12 SEM images of untreated buckypaper (a) and molded buckypaper (b) ${ }^{52}$.

Adapted from Electrochemical Soc Publisher. 
化去除表面富集的EP。经过等离子体处理, 可以 去除厚度为 $1 \mu \mathrm{m}$ 的表面树脂层, ASR降低了 $70 \%$ 。 Kim 等 ${ }^{17}$ 利用火焰灼烧压制成型的极板表面积聚 的PF, ASR有了显著的下降。

在极板表面铺设成型碳材料也可以有效降低 吸收富集树脂, 并降低表面接触电阻。Kim等 ${ }^{27}$ 将 混合有炭黑的PF浸渍连续碳纤维, 通过热轧将PF 与连续碳纤维部分结合并预固化, 随后在表面覆 盖碳纤维毡, 并进行热模压成型。试验结果表明, 当表面覆盖的碳毡厚度为 $80 \mu \mathrm{m}$ 时, ASR从 82 $\mathrm{m} \Omega \cdot \mathrm{cm}^{2}$ 下降至 $28 \mathrm{~m} \Omega \cdot \mathrm{cm}^{2}$, 但预固化工艺会使得 复合材料的抗弯强度下降 $20 \%$ 。 $\mathrm{Ha}$ 等利用石墨箔 吸收在成型过程中富集在极板表面的树脂, 石墨 䇴厚度分别为 $2 、 50 \mu \mathrm{m}$ 时, ASR分别为未覆盖石墨 䇴的 $14 \%$ 和 $10 \%$ 。

复合材料中填料与树脂之间通过摩擦、化 学键、范德华力等相互结合, 形成填料/树脂界 面 ${ }^{105-108}$ 。界面的性能对于复合材料的导热性、机 械性能等也有着直接的影响 109,110 。其中, 填料与 树脂的界面通常是复合材料发生机械损伤的诱 发位置。图 13 为混合有 $\mathrm{CF}$ 复合石墨极板断面的 SEM图像, 可以清晰的观察到 CF拔出后留下的孔 洞以及CF的断茬, 表明树脂与 CF 的结合强度相对 较低。Wang等对石墨烯进行官能团化处理, 并离 散在石蜡基质中, 并研究了跨石墨烯-石蜡界面 的热传输功能。研究结果显示, 石墨烯表面的官 能团能够直接影响到石墨与树脂间的热导率, 丁基 $\left(-\mathrm{C}_{4} \mathrm{H}_{9}\right)$ 能够显著降低石墨烯-石蜡的界面导热热 阻, 其次是甲基 $\left(-\mathrm{CH}_{3}\right)$ 、苯基 $\left(-\mathrm{C}_{6} \mathrm{H}_{5}\right)$ 和甲酰基
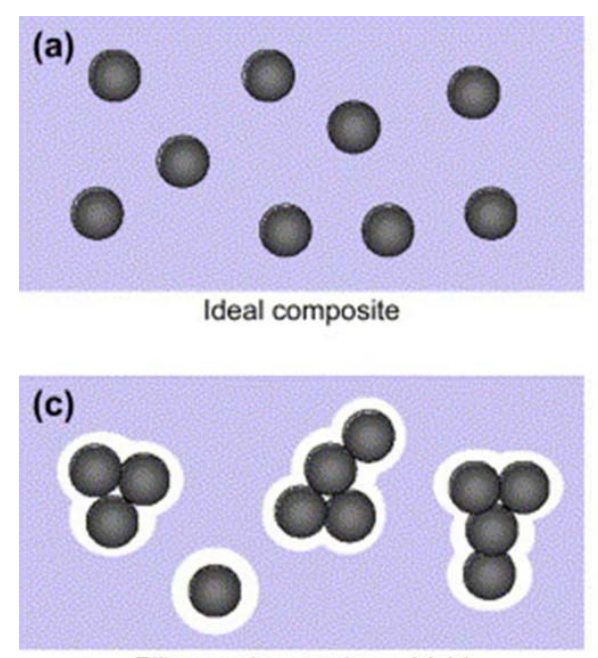

Filler agglomeration + Void

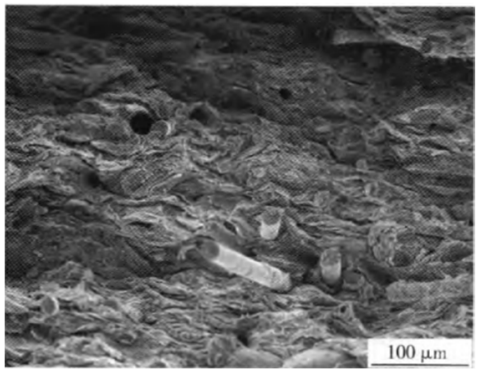

图13 含有 CF 的复合石墨极板断面SEM照片 ${ }^{37}$

Fig. 13 Fracture SEM photographs of composite bipolar plate with $\mathrm{CF}^{37}$.

Adapted from Acta Mater. Compos. Sin.

$(-\mathrm{COH})$, 而羧基 $(-\mathrm{COOH})$ 和羟基 $(-\mathrm{OH})$ 对于导 热性能的无明显影响111。

图14为填料在树脂基质中的离散以及填料/树 脂结合情况示意图。理想状态下, 填料应均匀离散 在基质中, 并且二者紧密地结合, 如图14a所示。若 填料/树脂相容性不佳, 则会在界面形成缝隙, 如 图14b所示。若填料出现连续团聚, 且填料/树脂相 容性不佳, 则会形成连续的缝隙, 如图14d所示。连 续的缝隙会成为气体渗透的通路, 极大地影响复 合材料的气密性 112,113 。Wang 等 ${ }^{114}$ 利用硫酸及硝酸 对MWCNT引入羧基, 随后利用乙二胺进行氨基官 能化, 并与聚乙烯胺(PVAm)混合制成纳米复合材 料薄膜。经过电镜观察发现, 未经官能团化的薄膜 存在明显的缝隙, 且均沿着MWCNT与PVAm的接 触界面如图15a所示, 官能团化后缝隙基本消失, 呈 现均匀的复合材料薄膜, 如图 $15 b$ 所示。可见, 填 料/树脂的界面性能是影响复合材料的气密性的重
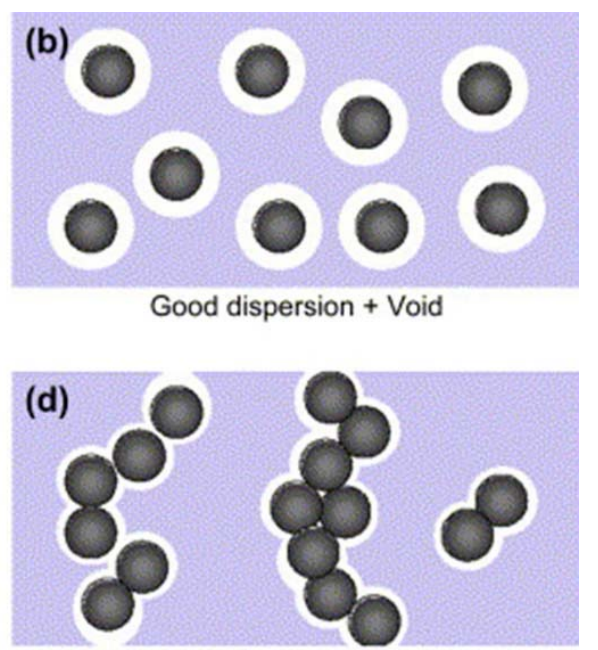

Filler agglomeration + Void channel

图14 含有填料的复合材料微观结构示意 ${ }^{113}$

Fig. 14 Schematic illustrations of possible morphologies of composites ${ }^{113}$. 


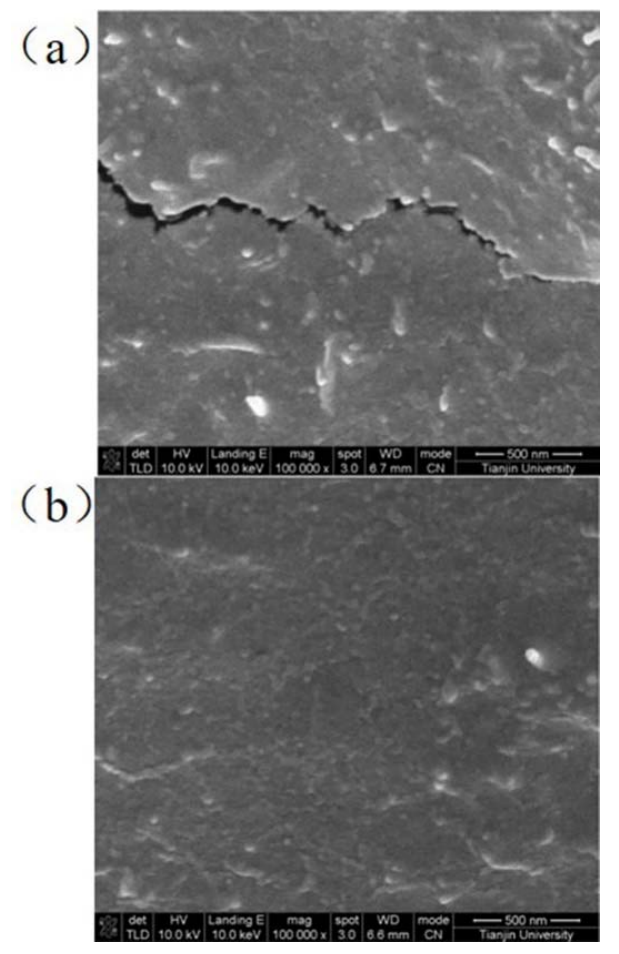

图15 纳米复合薄膜的SEM观测图像 114

Fig. 15 SEM cross-section morphologies of composite membranes ${ }^{114}$.

(a) MWCNT; (b) MWCNT-NH 2 . Adapted from Elsevier Science Publisher.

要影响因素, 官能团化对于提升气密性能也发挥 着重要的作用。

\section{4 功能层的制备工艺开发}

传统的复合石墨极板的性能优化主要依靠优 化石墨以及树脂的成分和配比, 然而这种优化方 式存在导电性能和抗弯强度难以同步优化的矛 盾。此外, 复合石墨极板厚度需要不断降低, 对于 复合材料性能的要求会进一步提高。采用具有特 殊结构或性能的功能层进行复合石墨极板的制作 就成为了一种十分具有研究价值的思路。

CFF由连续碳纤维编织构成, 在表面喷涂有 $\mathrm{EP}$ 溶液后可以通过热压固化在复合石墨极板中。 $\mathrm{CFF}$ 能够显著增强复合石墨极板的抗弯强度, 可以 达到150-195 MPa ${ }^{50,53}$ 。利用 $\mathrm{CFF}$ 制备极板还有利 于显著降低极板厚度 ${ }^{33,51}$ 。然而由于EP富集在CFF 表面, 会降低复合石墨极板的导电性能。Kim等 ${ }^{53}$ 将石墨粉混合在EP中, 制备成具有高导电性能的 预制层。随后依次将混合母料与 CFF堆叠在模具 中, 热压制成复合石墨极板。试验结果显示, 随着 EP中石墨粉含量的提升, 面内电导率和贯穿电导 率均呈现上升趋势, 但抗弯强度会逐渐下降。Kang 等 ${ }^{51}$ 通过在预浸料表面涂覆石墨粉, 降低复合石墨 材料的贯穿电导率, 并以石墨/树脂复合材料作为
极板表面层, 提高复合石墨极板的流道成型质量, 层压工艺如图16所示。试验结果显示, 预浸料表面 涂覆石墨粉能够有效降低复合石墨极板的贯穿电 导率, 且随着石墨粉的粒径从 $40 \mu \mathrm{m}$ 下降至 $6 \mu \mathrm{m}$, 贯穿电导率从 $10 \mathrm{~S} \cdot \mathrm{cm}^{-1}$ 提升至 $38 \mathrm{~S} \cdot \mathrm{cm}^{-1}$, 而未涂 覆石墨粉的预浸料贯穿电导率仅为 $4.8 \mathrm{~S} \cdot \mathrm{cm}^{-1}$ 。

Kim等 ${ }^{54}$ 在 CFF的一侧喷涂PF溶液, 并与石墨 䈃贴合, 通过辊压使 CFF与膨胀石墨䈃充分接触, 热模压固化定型, 制备成碳纤维/石墨箔分层式复 合石墨极板, 成型板材的厚度可以达到 $0.5 \mathrm{~mm}$, 截面的显微图像如图17所示。图17a显示PF的喷涂 量较少的情况, 碳纤维不能被完全润湿, 造成CFF 与石墨䈃不能紧密结合。喷涂量达到 $15 \mathrm{~g} \cdot \mathrm{m}^{-2}$ 以上 时, 可以保证CFF与石墨箔紧密接触, 如图 17b所 示。然而喷涂量不宜过大, 否则在成型过程中PF 会渗透到CFF与气体扩散层接触的接触面, 会显著 提高极板的ASR。在CFF表面清洁, 无PF包覆的情 况下, ASR可以保持在约 $20 \mathrm{~m} \Omega \cdot \mathrm{cm}^{2}$ 。经过对板材 气密性测试, 复合石墨极板具有良好的气密性, 文 中提出这是由于石墨箔气体渗透率极低。Minkook 等 ${ }^{45}$ 的研究结论也表明, $2 \mu \mathrm{m}$ 厚的石墨䈃即可显著 提升复合石墨极板的气密性能, 同时位于极板表 面的石墨䈃可以有效避免由于树脂富集。 $\mathrm{Yu}$ 等 49 的试验结论也表明, 石墨箔吸收表面富集的树脂 后, 复合石墨极板的贯穿面电阻大幅降低。相比于 未添加石墨簿的复合极板, 2 和 $50 \mu \mathrm{m}$ 的石墨簿分 别可以使ASR下降 $86 \%$ 和 $90 \%$ 。Du等 ${ }^{115}$ 将具有连续 导电结构的膨胀石墨板在浸渍在EP溶液中, 膨胀 石墨板中的气体完全排出后, 加热加压固化。当EP 溶液浓度达到 $40 \%$ 时，气密性可达到 $2 \times 10^{-6}$ $\mathrm{cm}^{3} \cdot \mathrm{s}^{-1} \cdot \mathrm{cm}^{-2} \cdot \mathrm{Pa}^{-1}$, 抗弯强度达到 $50 \mathrm{MPa}$, 同时 ASR 保持在 $20 \mathrm{~m} \Omega \cdot \mathrm{cm}^{2}$ 。

不仅CFF、石墨箔等成型碳材料能够作为复合

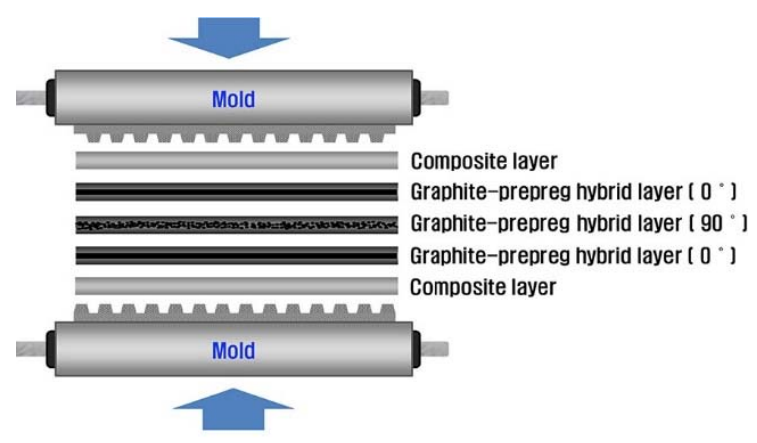

图16 预浸料-复合石墨极板层压成型工艺示意图 ${ }^{51}$

Fig. 16 Schematic of the compression molding process graphite-prepreg hybrid BPs ${ }^{\mathbf{5 1}}$.

Adapted from Elsevier Publisher. 
(a)

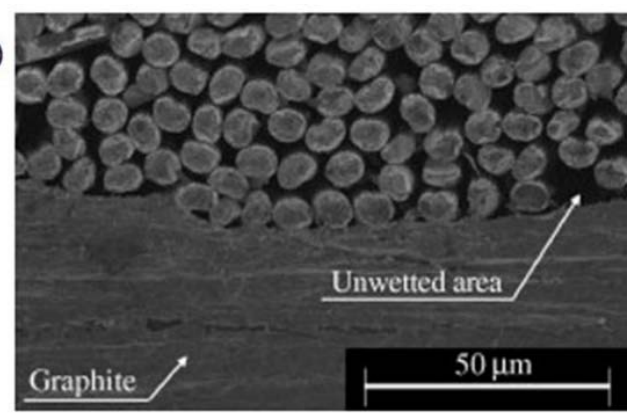

(b)

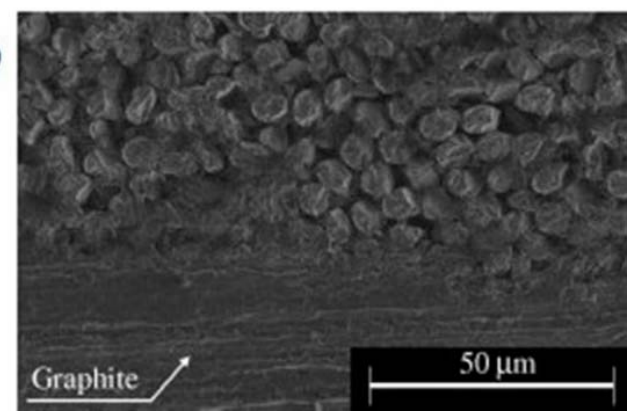

图17

不同PF喷涂量的碳纤维/石墨箔复合石墨极板的 截面SEM图像

Fig. 17 SEM images of cross section view of the carbon fabric/graphite hybrid composite bipolar plates with respect to areal densities of phenolic resin used.

(a) $6.6 \mathrm{~g} \cdot \mathrm{m}^{-2}$; (b) $17 \mathrm{~g} \cdot \mathrm{m}^{-2} 54$. Adapted from Elsevier Publisher.

石墨极板的功能层, 基于复合石墨材料制备工艺 生产的薄板、薄膜等材料也可作为复合石墨极板 的功能层。Kim等 ${ }^{116}$ 利用蒸发成型工艺制备复合石 墨薄膜, 然后堆叠层压制成用于液流电池的复合 石墨极板。石墨、CNT与PVB粘合剂溶液均匀混合 后延流在PET载体膜上, $80{ }^{\circ} \mathrm{C}$ 干燥后制成复合石 墨薄膜。随后将复合石墨薄膜堆叠后以 $40 \mathrm{MPa}$ 、 $80^{\circ} \mathrm{C}$ 的条件下热压, 形成厚度约为 $1 \mathrm{~mm}$ 的复合石 墨板。这种工艺的优势在于能够避免注塑成型过 程中填料分布不均, 以及高填料条件下复合材料 流动性不足等问题。蒸发成型工艺能够有效保证 导电填料在 $85 \%$ 的条件下的填料均匀分布, 且成型 极板ASR可以达到 $50 \mathrm{~m} \Omega \cdot \mathrm{cm}^{2}$ 。基于蒸发成型工 艺, 结合交流电场 ${ }^{92-95}$ 、磁场 ${ }^{96,97}$ 等填料定向工艺, 可以实现具有特定填料分布, 具有较高导电性能 的预制功能层。通过使用高导电功能层, 能够有效 降低复合极板的ASR ${ }^{117}$ 。

\section{5 总结与展望}

本文总结了目前应用于复合石墨极板制备的 常用导电填料和树脂材料, 制备原料的改性方法, 以及先进极板制备工艺。随着对于复合石墨极板 电导率、抗弯强度、成型厚度、气密性等性能要求
的进一步提高, 单纯依靠改进石墨/树脂的成分和 配比越来越难以实现各项性能的协同优化。同时, 结构对于复合石墨极板性能的影响也日渐得到了 重视: 导电填料的有序组织能够有效提升复合石 墨极板的导电性能; 去除极板表面富集的树脂, 有 利于大幅降低复合石墨极板的ASR; 石墨/树脂的 界面性能与极板的机械性能和气密性也有着直接 的联系; 依靠引入预制功能层, 能够实现对于极板 各方面性能的分别优化。

可见, 配方的优化与先进制备工艺的结合是 协同优化各项性能的重要研究方法。基于对现有 复合石墨极板相关研究的总结和对比, 未来复合 石墨极板的开发可能会从以下几个方面展开:

(1)对于传导网络进行系统的分析与研究。以 构建更高效的传导网络为研究目标, 通过多种填 料协同使用, 开发新型加工工艺, 使用成型碳基材 料等方法, 优化复合石墨极板之中的导电结构;

(2)优化石墨/树脂的界面性能。依靠对于石 墨、树脂的改性处理, 提高石墨与树脂的界面性 能, 提升复合石墨极板的机械性能与气密性。由于 改性处理通常会削弱石墨的导电性能, 因此界面 性能与导电性能的平衡值得详细探讨;

(3)开发不同功能层复合石墨极板及其制备 工艺。依托先进的复合石墨材料的制备工艺, 制备 具有高强度、高导电性、高气密性等的预制功能 层, 并将其结合到复合石墨极板的制备工艺中。依 托各功能层性能的分别优化, 实现复合石墨极板 整体性能的优化。

\section{References}

(1) Mah, A. X. Y.; Ho, W. S.; Bong, C. P. C.; Hassim, M. H.; Liew, P. Y.; Asli, U. A.; Kamaruddin, M. J.; Chemmangattuvalappil, N. G. Int. J. Hydrogen Energ. 2019, 44, 5661.

doi: 10.1016/j.ijhydene.2019.01.077

(2) Liu, B.; Liu, S.; Guo, S.; Zhang, S. Int. J. Hydrogen Energ. 2020, 42, 1385. doi: 10.1016/j.ijhydene.2019.11.056

(3) Garcia, D. A.; Barbanera, F.; Cumo F.; Matteo U. D.; Nastasi, B. Energies 2016, 9, 963. doi: 10.3390/en9110963

(4) Sinigaglia, T.; Lewiski, F.; Martins, M. E. S.; Siluk, J. C. M. Int. J. Hydrogen Energ. 2017, 42, 24597.

doi: 10.1016/j.ijhydene.2017.08.063

(5) Liu, J.; Zhong, C. F. Energy of China 2019, 41, 32. [刘坚, 钟财富. 中国能源, 2019, 41, 32.] doi: 10.3969/j.issn.1003-2355.2019.02.007

(6) Badea, G.; Naghiu, G. S.; Giurca, I.; Aşchilean, I.; Megyesi, E. Energy Procedia 2017, 112, 418. doi: 10.1016/j.egypro.2017.03.1097 (7) Vincent, I.; Bessarabov, D. Renew Sust. Energ. Rev. 2018, 81, 1690. 
doi: 10.1016/j.rser.2017.05.258

(8) Møllera, K. T.; Jensena, T. R.; Akibab, E.; Li, H. W. Prog. Nat. Sci. Mater. 2017, 27, 34. doi: 10.1016/j.pnsc.2016.12.014

(9) Marchenko, O. V.; Solomin, S. V. Int. J. Hydrogen Energ. 2017, 42, 9361. doi: 10.1016/j.ijhydene.2017.02.076

(10) Budak, Y.; Devrim, Y. Energ. Convers. Manage 2018, 160, 486. doi: 10.1016/j.enconman.2018.01.077

(11) Li, Y.; Yang, J.; Song, J. Renew. Sust. Energ. Rev. 2017, 67, 160. doi: 10.1016/j.rser.2016.09.030

(12) Li, J. C.; Wang, Q.; Jiang, R.; Wu, A. M.; Lin, G. Q.; Dong, C. Mater. Rev. 2018, 32, 2584. [李俊超, 王清, 蒋锐, 吴爱民, 林国强, 董闯. 材料导报, 2018, 32, 2584.] doi: 10.11896/j.issn.1005-023X.2018.15.008

(13) Dafalla, A. M.; Jiang, F. Int. J. Hydrogen Energ. 2018, 43, 2327. doi: 10.1016/j.ijhydene.2017.12.033

(14) Radzuan, N. A. M.; Sulong, A. B.; Somalu, M. R. Sains. Malays 2019, 48, 669. doi: 10.17576/jsm-2019-4803-21

(15) Leng, Y.; Ming, P.; Yang, D.; Zhang, C. J. Power Sources 2020, 451, 227783. doi: 10.1016/j.jpowsour.2020.227783

(16) Liu, F.; Yi, B.; Xing, D.; Yu, J.; Zhang, H. J. Membrane Sci. 2003, 212, 213. doi: 10.1016/S0376-7388(02)00503-3

(17) Kim, M.; Lim, J. W.; Kim, K. H.; Lee, D. G. Compos. Struct. 2013, 96, 569. doi: 10.1016/j.compstruct.2012.09.017

(18) Singh, R. S.; Gautam, A.; Rai, V. Front. Mater. Sci. 2019, 13, 217. doi: 10.1007/s11706-019-0465-0

(19) Stein, T.; Ein-Eli, Y. Energy Technol. 2020, 8, 2000007. doi: $10.1002 /$ ente. 202000007

(20) Wang, H.; Turner, J. A. Fuel Cells 2010, 10, 510. doi: $10.1002 /$ fuce. 200900187

(21) Hermann, A.; Chaudhuri, T.; Spagnol, P. Int. J. Hydrogen Energ. 2005, 30, 1297. doi: 10.1016/j.ijhydene.2005.04.016

(22) Li, Y.; Jia, X.; Zhang, W.; Fang, C.; Wang, X.; Qin, F.; Yamaura, S.; Yokoyama, Y. Metall. Mater. Trans. A 2013, 45, 2393. doi: 10.1007/s11661-013-2071-6

(23) Liang, P.; Xu, H. F.; Liu, M.; Lu, L.; Fu, J. Acta Phys. -Chim. Sin. 2010, 26, 595. [梁鹏, 徐洪峰, 刘明, 卢璐, 傅杰. 物理化学学报, 2010, 26, 595.] doi: 10.3866/PKU.WHXB20100329

(24) Wilberforce, T.; Ijaodola, O.; Ogungbemi, E.; Khatib, F. N.; Leslie, T.; El-Hassan, Z.; Thomposon, J.; Olabi, A. G. Renew. Sust. Energ. Rev. 2019, 113, 109286. doi: 10.1016/j.rser.2019.109286

(25) Vlaskin, M. S.; Grigorenko, A. V.; Shkolnikov, E. I.; Ilyukhin, A. S. Surf. Rev. Lett. 2019, 26, 1950038. doi: 10.1142/S0218625X19500380

(26) Qian, Y.; Xu, J. Acta Phys. -Chim. Sin. 2015, 31, 291. [钱阳, 徐江. 物理化学学报, 2015, 31, 291.] doi: 10.3866/PKU.WHXB201411262

(27) Kim, M.; Lim, J. W.; Lee, D. G. Compos. Struct. 2015, 119, 630. doi: 10.1016/j.compstruct.2014.09.010

(28) Ji, S.; Hwang, Y. S.; Park, T.; Lee, Y. H.; Paek, J. Y.; Chang, I.; Lee, M. H.; Cha, S. W. Int. J. Precis. Eng. Man. 2012, 13, 2183. doi: 10.1007/s12541-012-0289-7

(29) Tripathi, B. P.; Shahi, V. K. Prog. Polym. Sci. 2011, 36, 945. doi: 10.1016/j.progpolymsci.2010.12.005

(30) Clingerman, M. L.; King, J. A.; Schulz, K. H.; Meyers, J. D. J. Appl. Polym. Sci. 2002, 83, 1341. doi: 10.1002/app.10014

(31) Zakaria, M. Y.; Sulong, A. B.; Sahari, J.; Suherman, H. Compos. Pt. B-Eng. 2015, 83, 75. doi: 10.1016/j.compositesb.2015.08.034

(32) Phuangngamphan, M.; Okhawilai, M.; Hiziroglu, S.; Rimdusit, S. J. Appl. Polym. Sci. 2019, 136, 47183. doi: 10.1002/app.47183

(33) Kim, M.; Lim, J. W.; Lee, D. G. Compos. Struct. 2018, 189, 79. doi: 10.1016/j.compstruct.2018.01.067

(34) Dweiri, R.; Suherman, H.; Sulong, A. B.; Al-Sharab, J. F. Sci. Eng. Compos. Mater. 2018, 25, 1177. doi: 10.1515/secm-2017-0122

(35) Akhtar, M. N.; Sulong, A. B.; Umer, A.; Yousaf, A. B.; Khan, M. A. Ceram. Int. 2018, 44, 14457. doi: 10.1016/j.ceramint.2018.05.059

(36) Radzuan, N. A. M.; Zakaria, M. Y.; Sulong, A. B.; Sahari, J. Compos. Pt. B-Eng. 2017, 110, 153. doi: 10.1016/j.compositesb.2016.11.021

(37) Chen, H.; Liu, H. B.; Xia, X. H.; Yang, L.; He, Y. D. Acta Mater. Compos. Sin. 2015, 32, 744. [陈慧，刘洪波，夏笑虹，杨丽，何月

德. 复合材料学报, 2015, 32, 744.] doi: 10.13801/j.cnki.fhclxb.201503.008

(38) Suherman, H.; Sulong, A. B.; Sahari, J. Ceram. Int. 2013, 39, 1277. doi: 10.1016/j.ceramint.2012.07.059

(39) Antunes, R. A.; Oliveira, M. C. L. D.; Ett, G.; Ett, V. J.Power Sources 2011, 196, 2945. doi: 10.1016/j.jpowsour.2010.12.041

(40) Petrach, E.; Abu-Isa, I.; Xia, W. J. Compos. Mater. 2010, 44, 1665. doi: $10.1177 / 0021998309357088$

(41) Diaz, J.; Rigail-Cedeño, A.; Barzola-Monteses, J.; Espinoza-Andaluz, M. Energy Procedia 2019, 158, 1502. doi: 10.1016/j.egypro.2019.01.358

(42) Jiang, X.; Drzal, L. T. J. Power Sources 2012, 218, 297. doi: 10.1016/j.jpowsour.2012.07.001

(43) Fukushima, H.; Drzal, L. T.; Rook, B. P.; Rich, M. J. J. Therm. Anal. Calorim. 2006, 85, 235. doi: 10.1007/s10973-005-7344-x

(44) Biswas, S.; Fukushima, H.; Drzal, L. T. Compos. Pt. A-Appl. Sci. Manuf. 2011, 42, 371. doi: 10.1016/j.compositesa.2010.12.006

(45) Kim, M.; Yu, H. N.; Lim, J. W.; Lee, D. G. Int. J. Hydrogen Energ. 2012, 37, 4300. doi: 10.1016/j.ijhydene.2011.11.125

(46) Lee, H. E.; Han, S. H.; Song, S. A.; Kim, S. S. Compos. Struct. 2015, 134, 44. doi: 10.1016/j.compstruct.2015.08.037

(47) Naji, A.; Krause, B.; Pötschke, P.; Ameli, A. Smart Mater. Struct. 2019, 28, 064004. doi: 10.1088/1361-665X/ab19cb

(48) Wei, T.; Song, L.; Zheng, C.; Wang, K.; Yan, J.; Shao, B.; Fan, Z. J. 
Mater. Lett. 2010, 64, 2376. doi: 10.1016/j.matlet.2010.07.061

(49) Yu, H. N.; Lim, J. W.; Suh, J. D.; Lee, D. G. J. Power Sources 2011, 196, 9868. doi: 10.1016/j.jpowsour.2011.06.102

(50) Hwang, I. U.; Yu, H. N.; Kim, S. S.; Lee, D. G.; Suh, J. D.; Lee, S. H.; Ahn, B. K.; Kim, S. H.; Lim, T. W. J. Power Sources 2008, 184, 90. doi: 10.1016/j.jpowsour.2008.05.088

(51) Kang, K.; Park, S.; Jo, A.; Lee, K.; Ju, H. Int. J. Hydrogen Energ. 2017, 42, 1691. doi: 10.1016/j.ijhydene.2016.05.027

(52) Yao, K; Adams, D. L.; Hao A.; Zheng, J. P.; Liang, R. ECS Trans. 2017, 77, 1303. doi: 10.1149/07711.1303ecst

(53) Kim, J. W.; Kim, N. H.; Kuilla, T.; Kim, T. J.; Rhee, K. Y.; Lee, J. H. J. Power Sources 2010, 195, 5474. doi: 10.1016/j.jpowsour.2010.03.083

(54) Kim, K. H.; Lim, J. W.; Kim, M.; Lee, D. G. Compos. Struct. 2013, 98, 103. doi: 10.1016/j.compstruct.2012.10.043

(55) Di, J. T.; Hu, D. M.; Chen, H. Y.; Yong, Z. Z.; Chen, M. H.; Feng, Z. H.; Zhu, Y. T.; Li, Q. W. ACS Nano 2012, 6, 5457. doi: $10.1021 / \mathrm{nn} 301321 \mathrm{j}$

(56) Yao, K.; Adams, D.; Hao, A.; Zheng, J. P.; Liang, Z. Y.; Nguyen, N. Energ. Fuel 2017, 31, 14320. doi: 10.1021/acs.energyfuels.7b02678

(57) Fiedler, B.; Gojny, F. H.; Wichmann, M. H. G.; Nolte, M. C. M.; Schulte, K. J. Compos. Sci. Technol. 2006, 66, 3115. doi: 10.1016/j.compscitech.2005.01.014

(58) Sham, M. L.; Kim, J. K. Carbon 2006, 44, 768. doi: 10.1016/j.carbon.2005.09.013

(59) Yin, Q.; Sun, K. N.; Li, A. J.; Shao, L.; Liu, S. M.; Sun, C. J Power Sources 2008, 175, 861. doi: 10.1016/j.jpowsour.2007.10.013

(60) Athmouni, N.; Mighri, F.; Elkoun, S. Polym. Advan. Technol. 2018, 29, 294. doi: $10.1002 /$ pat.4114

(61) Lee, M. H.; Kim, H. Y.; Kim, J.; Han, J. T.; Lee, Y. S.; Woo, J. S. Carbon Lett. 2019, 30, 345. doi: 10.1007/s42823-019-00103-2

(62) Liao, S. H.; Weng, C. C.; Yen, C. Y.; Hsiao, M. C.; Ma, C. C. M.; Tsai, M. C.; Su, A.; Yen, M. Y.; Lin, Y. F.; Liu, P. L. J. Power Sources 2010, 195, 263. doi: 10.1016/j.jpowsour.2009.06.064

(63) Li, J.; Vaisman, L.; Marom, G.; Kim, J. K. Carbon 2007, 45, 744. doi: $10.1016 /$ j.carbon.2006.11.031

(64) Matsumoto, R.; Okabe, Y. Synthetic Met. 2016, 222, 351. doi: 10.1016/j.synthmet.2016.11.020

(65) Kalaitzidou, K.; Fukushima, H.; Drzal, L. Materials 2010, 3, 1089. doi: $10.3390 / \mathrm{ma} 3021089$

(66) Taherian, R. Compos. Sci. Technol. 2016, 123, 17. doi: 10.1016/j.compscitech.2015.11.029

(67) Radzuan, M.; Afiqah, N.; Sulong, A. B.; Sahari, J. Int. J. Hydrogen Energ. 2017, 42, 9262. doi: 10.1016/j.ijhydene.2016.03.045

(68) Mclachlan, D. S.; Blaszkiewicz, M.; Newnham, R. E. J. Am. Ceram. Soc. 1990, 73, 2187. doi: 10.1111/j.1151-2916.1990.tb07576.x
(69) Mamunya, E. P.; Davidenko, V. V.; Lebedev, E. V. Compos. Interface 2012, 4, 169. doi: 10.1163/156855497x00145

(70) Nielsen, L. E. J. Ind. Eng. Chem. 1974, 13, 17. doi: 10.1021/i160049a004

(71) Lee, D.; Lee, D. G. J. Power Sources 2016, 327, 119. doi: 10.1016/j.jpowsour.2016.07.045

(72) Ouyang, T.; Yin, S. F.; Xie, Z. Y.; Gao, P. P.; Tao, T.; Huang, Q. Z. Acta Mater. Compos. Sin. 2018, 35, 2950. [欧阳涛, 尹绍峰, 谢志勇, 高平平, 陶韬, 黄启忠. 复合材料学报, 2018, 35, 2950.] doi: 10.13801/j.cnki.fhclxb.20180316.002

(73) San, F. G. B.; Tekin, G. Int. J. Energ. Res. 2013, 37, 283. doi: 10.1002/er.3005

(74) Dweiri, R.; Sahari, J. J. Power Sources 2007, 171, 424. doi: 10.1016/j.jpowsour.2007.05.106

(75) Mahyoedin, Y.; Sahari, J.; Mukhtar, A.; Mohammad, N.; Suryadimal. MATEC Web of Conferences 2018, 248, 01007. doi: 10.1051/matecconf/201824801007

(76) Lim, J. W.; Kim, M.; Yu, Y. H.; Lee, D. G. Compos. Struct. 2014, 118, 519. doi: 10.1016/j.compstruct.2014.08.011

(77) Martins, J. N.; Kersch, M.; Altstädt, V.; Oliveira, R. V. B. Polym. Test. 2013, 32, 1511. doi: 10.1016/j.polymertesting.2013.10.001

(78) Ansari, S.; Giannelis, E. P. J. Polym. Sci. Pol. Phy. 2009, 47, 888. doi: $10.1002 /$ polb.21695

(79) Liao, S. H.; Yen, C. Y.; Weng, C. C.; Lin, Y. F.; Ma, C. C. M.; Yang, C. H.; Tsai, M. C.; Yen, M. Y.; Hsiao, M. C.; Lee, S .J. J. Power Sources 2008, 185, 1225. doi: 10.1016/j.jpowsour.2008.06.097

(80) Adloo, A.; Sadeghi, M.; Masoomi, M.; Pazhooh, H. N. Renew. Energ. 2016, 99, 867. doi: 10.1016/j.renene.2016.07.062

(81) Caglar, B.; Fischer, P.; Kauranen, P.; Karttunen, M.; Elsner, P. J. Power Sources 2014, 256, 88. doi: 10.1016/j.jpowsour.2014.01.060

(82) Lee, M. H.; Kim, H. Y.; Oh, S. M.; Kim, B. C.; Bang, D.; Han, J. T.; Woo, J. S. Int. J. Hydrogen Energ. 2018, 43, 21918. doi: 10.1016/j.ijhydene.2018.09.104

(83) Hopmann, C.; Windeck, C.; Cohnen, A.; Onken, J.; Krause, B.; Pötschke, P.; Hickmann, T. AIP Conference Proceedings 2016, 1779, 030017. doi: $10.1063 / 1.4965487$

(84) Yeetsorn, R.; Fowler, M.; Tzoganakis, C.; Yuhua, W.; Taylor, M. Macromol. Symp. 2008, 264, 34. doi: 10.1002/masy.200850406

(85) Alo, O. A.; Otunniyi, I. O.; Pienaar, H. Polym. Compos. 2020, 41, 3364. doi: $10.1002 /$ pc. 25625

(86) Simaafrookhteh, S.; Khorshidian, M.; Momenifar, M. Int. J. Hydrogen Energ. 2020, 45, 14119. doi: 10.1016/j.ijhydene.2020.03.105

(87) Park, H. J.; Woo, J. S.; Kim, S. H.; Park, K. S.; Park, S. H.; Park, S. Y. Macromol. Res. 2019, 27, 1161. doi: 10.1007/s13233-019-7156-7 (88) Liao, W.; Jiang, F.; Zhang, Y.; Zhou, X.; He, Z. Renew. Energ. 2020, 
152, 1310. doi: 10.1016/j.renene.2020.01.155

(89) Kim, S. H.; Woo, J. S.; Park, S. Y. Macromol. Res. 2020. doi: $10.1007 / \mathrm{s} 13233-020-8140-\mathrm{y}$

(90) Radzuan, M.; Afiqah, N.; Sulong, A. B.; Somalu, M. R.; Abdullah, A. T.; Husaini, T.; Rosli, R. E.; Majlan, E. H.; Rosli, M. I. Int. J. Hydrogen Energ. 2019, 44, 30618. doi: 10.1016/j.ijhydene.2019.01.063

(91) Huang, J.; Rodrigue, D. Mater. Design 2014, 55, 653. doi: 10.1016/j.matdes.2013.10.039

(92) Martin, C. A.; Sandler, J. K. W.; Windle, A. H.; Schwarz, M. K.; Bauhofer, W.; Schulte, K.; Shaffer, M. S. P. Polymer 2005, 46, 877. doi: 10.1016/j.polymer.2004.11.081

(93) Senis, E. C.; Golosnoy, I. O.; Andritsch, T.; Dulieu-Barton, J. M.; Thomsen, O. T. Polym. Compos. 2020, 41,3510. doi: $10.1002 /$ pc. 25637

(94) Gupta, P.; Rajput, M.; Singla, N.; Kumar, V.; Lahiri, D. Polymer 2016, 89, 119. doi: 10.1016/j.polymer.2016.02.025

(95) Wang, Q.; Dai, J.; Li, W.; Wei, Z.; Jiang, J. Compos. Sci. Technol. 2008, 68, 1644. doi: 10.1016/j.compscitech.2008.02.024

(96) Ma, C. G.; Liu, H. Y.; Du, X. S.; Mach, L. T.; Xu, F.; Mai, Y. W. Compos. Sci. Technol. 2015, 114, 126. doi: 10.1016/j.compscitech.2015.04.007

(97) Tanabi, H.; Erdal, M. Results Phys. 2019, 12, 486. doi: 10.1016/j.rinp.2018.11.081

(98) Liu, N.; Liu, Y. Z.; Zhao, Y. L.; Liu, Y. T.; Lan, Q.; Qin, J.; Song, Z. P.; Zhan, H. ACS Appl. Mater. Inter. 2019, 11, 467264. doi: $10.1021 /$ acsami.9b15462

(99) Zabihi, O.; Shafei, S.; Fakhrhoseini, S. M.; Ahmadi, M.; Nazarloo, H. A.; Stanger, R.; Tran, Q. A.; Lucas, J.; Wall, T.; Naebe, M. Materials 2019, 12, 1281. doi: 10.3390/ma12081281

(100) Sen, R.; Zhao, B.; Perea, D.; Itkis, M. E.; Hu, H.; Love, J.; Bekyarova, E.; Haddon, R. C. Nano Lett. 2004, 4, 459. doi: $10.1021 / \mathrm{n} 1035135 \mathrm{~s}$

(101) Avasarala, B.; Haldar, P. J. Power Sources 2009, 188, 225. doi: 10.1016/j.jpowsour.2008.11.063

(102) Lee, D.; Lee, D. G. Compos. Struct. 2016, 140, 77. doi: 10.1016/j.compstruct.2015.12.066

(103) Lee, D.; Lee, D. G.; Lim, J. W. J. Intel. Mat. Syst. Str. 2017, 29,
3386. doi: $10.1177 / 1045389 \times 17708345$

(104) Yu, H. N.; Lim, J. W.; Kim, M. K.; Lee, D. G. Compos. Struct. 2012, 94, 1911. doi: 10.1016/j.compstruct.2011.12.024

(105) Li, B.; Liu, D.; Li, G.; Yang, X. J. Mater. Sci. 2018, 53, 15939. doi: 10.1007/s10853-018-2753-y

(106) Ang, K. K.; Ahmed, K. S. Compos. Pt. B-Eng. 2013, 50, 7. doi: 10.1016/j.compositesb.2013.01.016

(107) Xu, F.; Liu, H. Y.; Du, X. Polumers 2018, 10, 863. doi: $10.3390 /$ polym 10060683

(108) Naya, F.; Molina-Aldareguia, J.; Lopes, C. S.; González, C.; Llorca, J. J. Miner. Met. Mater. Soc. 2016, 69, 13. doi: 10.1007/s11837-016-2128-2

(109) Paul, R.; Dai, L. Compos. Interface 2018, 25, 539. doi: $10.1080 / 09276440.2018 .1439632$

(110) Xiong, Y. H.; Wu, H.; Gao, J. S.; Chen, W.; Zhang, J. C.; Yue, Y. N. Acta Phys. -Chim. Sin. 2019, 35, 1150. [熊扬恒, 吴昊, 高建树, 陈 文, 张景超, 岳亚楠. 物理化学学报, 2019, 35, 1150.] doi: 10.3866/PKU.WHXB201901002

(111) Wang, Y.; Zhan, H. F.; Xiang, Y.; Yang, C.; Wang, C. M.; Zhang, Y. Y. J. Phys. Chem. C 2015, 119, 12731 doi: 10.1021/acs.jpcc.5b02920

(112) Jia, M. D.; Pememann, K. V.; Behlmg, R. D. J. Membrane Sci. 1992, 73, 199. doi: 10.1016/0376-7388(92)80122-Z

(113) Takahashi, S.; Paul, D. R. Polyer 2006, 47, 7519. doi: 10.1016/j.polymer.2006.08.029

(114) Wang, M.; Wang, Z.; Li, N.; Liao, J. Y.; Zhao, S.; Wang, J. X.; Wang, S. C. J. Membrane Sci. 2015, 495, 252. doi: 10.1016/j.memsci.2015.08.019

(115) Du, C.; Ming, P.; Hou, M.; Fu, J.; Fu, Y.; Luo, X.; Shen, Q.; Shao, Z.; Yi, B. J. Power Sources 2010, 195, 5312. doi: 10.1016/j.jpowsour.2010.03.005

(116) Kim, M. Y.; Choi, S. W.; Boo, S. J.; Lee, J. H.; Noh, H. S.; Kim, H. S. J. Nanosci. Nanotechnol. 2015, 15, 8055. doi: 10.1166/jnn.2015.11245

(117) Guo, J.; Zhang, Q. J.; Gao, L.; Zhong, W. H.; Sui, G.; Yang, X. P. Compos. Pt. A-Appl. Sci. Manuf. 2017, 95, 294. doi: 10.1016/j.compositesa.2017.01.021 